\title{
Metabolic profiling indicates impaired pyruvate dehydrogenase function in myalgic encephalopathy/chronic fatigue syndrome
}

Øystein Fluge, ${ }^{1}$ Olav Mella, ${ }^{1,2}$ Ove Bruland, ${ }^{1,3}$ Kristin Risa, ${ }^{1}$ Sissel E. Dyrstad, ${ }^{4}$ Kine Alme, ${ }^{1}$ Ingrid G. Rekeland, ${ }^{1}$ Dipak Sapkota, ${ }^{1}$ Gro V. Røsland, ${ }^{4}$ Alexander Fosså, ${ }^{5}$ Irini Ktoridou-Valen, ${ }^{1}$ Sigrid Lunde, ${ }^{1}$ Kari Sørland, ${ }^{1}$ Katarina Lien, ${ }^{6}$ Ingrid Herder, ${ }^{6}$ Hanne Thürmer, ${ }^{7}$ Merete E. Gotaas, ${ }^{8}$ Katarzyna A. Baranowska, ${ }^{8}$ Louis M.L.J. Bohnen, ${ }^{9}$ Christoph Schäfer, ${ }^{9}$ Adrian McCann, ${ }^{10}$ Kristian Sommerfelt, ${ }^{11}$ Lars Helgeland, ${ }^{12}$ Per M. Ueland, ${ }^{2,10}$ Olav Dahl, ${ }^{1,2}$ and Karl J. Tronstad ${ }^{4}$

'Department of Oncology and Medical Physics, Haukeland University Hospital, Bergen, Norway. ${ }^{2}$ Department of Clinical Science, University of Bergen, Bergen, Norway. ${ }^{3}$ Department of Medical Genetics and Molecular Medicine, Haukeland University Hospital, Bergen, Norway. ${ }^{4}$ Department of Biomedicine, University of Bergen, Bergen, Norway. ${ }^{5}$ Department of Oncology, Norwegian Radium Hospital, Oslo University Hospital, Oslo, Norway. ${ }^{6} \mathrm{CFS} / \mathrm{ME}$ Center, Division of Medicine, Oslo University Hospital, Oslo, Norway. ${ }^{7}$ Telemark Hospital, Department of Medicine, Notodden, Norway. ${ }^{8}$ Department of Pain and Complex Disorders, St. Olav's Hospital, Trondheim, Norway. ${ }^{\circ}$ Division of Rehabilitation Services, University Hospital of Northern Norway, Tromsø, Norway. ${ }^{10}$ Bevital AS, Bergen, Norway. ${ }^{11}$ Department of Pediatrics and ${ }^{12}$ Department of Pathology, Haukeland University Hospital, Bergen, Norway.

Myalgic encephalopathy/chronic fatigue syndrome (ME/CFS) is a debilitating disease of unknown etiology, with hallmark symptoms including postexertional malaise and poor recovery. Metabolic dysfunction is a plausible contributing factor. We hypothesized that changes in serum amino acids may disclose specific defects in energy metabolism in ME/CFS. Analysis in 200 ME/CFS patients and 102 healthy individuals showed a specific reduction of amino acids that fuel oxidative metabolism via the TCA cycle, mainly in female ME/CFS patients. Serum 3-methylhistidine, a marker of endogenous protein catabolism, was significantly increased in male patients. The amino acid pattern suggested functional impairment of pyruvate dehydrogenase (PDH), supported by increased mRNA expression of the inhibitory PDH kinases 1, 2, and 4; sirtuin 4; and PPAR $\delta$ in peripheral blood mononuclear cells from both sexes. Myoblasts grown in presence of serum from patients with severe ME/CFS showed metabolic adaptations, including increased mitochondrial respiration and excessive lactate secretion. The amino acid changes could not be explained by symptom severity, disease duration, age, BMI, or physical activity level among patients. These findings are in agreement with the clinical disease presentation of ME/CFS, with inadequate ATP generation by oxidative phosphorylation and excessive lactate generation upon exertion.

University Hospital has patents and pending patent applications on the issue of $\mathrm{B}$ cell depletion therapy for myalgic encephalopathy/chronic fatigue syndrome, a treatment principle mentioned in the discussion of the article. Family members of W02009083602 A1 are pending and some of them are granted, including US 7.914.785. Øystein Fluge and Olav Mella are named as inventors.

Submitted: July 5, 2016 Accepted: November 15, 2016 Published: December 22, 2016

Reference information: JCI Insight. 2016;1(21):e89376. doi:10.1172/jci.insight.89376.

\section{Introduction}

Myalgic encephalopathy/chronic fatigue syndrome (ME/CFS) is a poorly understood disease of unknown etiology that affects $0.1 \%-0.2 \%$ of the population, according to Canadian consensus criteria (1). Compared with many other chronic diseases, patients with ME/CFS have a lower quality of life, with major implications for patients and their families and for society $(2,3)$. Recent research suggests that ME/CFS is associated with changes in fundamental processes of energy metabolism $(4,5)$. Importantly, such metabolic changes may arise from dysregulated physiological response mechanisms that may be relevant in ME/CFS, such as immune activation, inflammation, and receptor-mediated signaling $(6,7)$. However, there are no consistent data indicating a common metabolic defect that could explain the symptoms in these patients. Identification of responsible mechanisms is urgent in order to understand the disease pathophysiology and for the development of clinical strategies to diagnose and treat the patients. 
The main symptoms of ME/CFS are fatigue, postexertional malaise, and lack of adequate restitution after rest or sleep, accompanied by cognitive disturbances and sensory hypersensitivity, including pain. The intensity of the symptoms is increased by exertion (8). Patients frequently suffer from additional symptoms ascribed to the autonomic nervous system or cardiovascular system, such as dizziness and palpitations, cold hands and feet, disturbed perceived body temperature, thirst, irritable bowel, and urinary urgency. Immune symptoms include recurrent sore throat and tender lymph nodes (1). In several studies, ME/CFS patients demonstrated reduced functional capacity in repeated cardiopulmonary exercise tests compared with healthy controls (9-11). These observations suggest that the systemic exertion intolerance in ME/CFS may, at least in part, involve a switch to anaerobic glycolysis, with generation of lactate at a significantly lower workload threshold than that observed for healthy subjects. Increased lactate levels have been found in the cerebrospinal fluid of ME/CFS patients $(12,13)$. However, defects in glucose utilization and lactate production are not evident based on routine blood sample analyses, possibly because the sampling is performed under resting conditions without preceding physical exercise.

Changes in serum (or plasma) concentrations of certain amino acids have been reported in ME/ CFS patients. A study of serum amino acid levels in 11 CFS patients and 10 healthy controls, using nuclear magnetic resonance spectroscopy, showed significantly lower levels of the amino acids glutamine (Gln) and ornithine in patients, indicating a possible disturbance in amino acid and nitrogen metabolism (4). Further, the same authors found significantly reduced serum levels of glutamate (Glu) and phenylalanine (Phe) in 34 female ME/CFS patients compared with 25 healthy females, and they proposed that reduced glucose oxidation led to increased use of amino acids as substrate for the TCA cycle, causing a decrease in serum Glu (14). In another study, significant reductions in the amounts of several amino acids and metabolites were observed in urine of $100 \mathrm{ME} / \mathrm{CFS}$ patients, compared with 82 healthy controls (15). These data support the presence of a metabolic defect in these patients, but the specific cause has not been identified.

Anaerobic threshold and recovery time after exercise depend on rates of lactate production and clearance. Lactate is made from pyruvate, mostly arising from glucose catabolism via the glycolytic pathway or from breakdown of certain amino acids. Under normal aerobic conditions, pyruvate is transported into mitochondria, where it is converted to acetyl-CoA by the pyruvate dehydrogenase $(\mathrm{PDH})$ complex. In addition, acetyl-CoA is produced independently of $\mathrm{PDH}$, via degradation of fatty acids and ketogenic amino acids. The acetyl-CoA formed in these processes is further oxidized in the TCA cycle and thereby serves to fuel mitochondrial respiration and adenosine triphosphate (ATP) production by oxidative phosphorylation. Under anaerobic conditions, when lack of oxygen prohibits mitochondrial respiration, pyruvate builds up in cytosol, which leads to increased production and cellular excretion of lactate. Mitochondrial dysfunction leads to both excessive lactate production and a deficient supply of ATP (16) and has been suggested to play a role in ME/CFS (17). PDH functions as a gateway in oxidative metabolism by coordinating the breakdown of 2- and 3-carbon energy substrates derived from carbohydrates, fats, and amino acids (18). A reduction in PDH enzymatic activity may lead to accumulation of pyruvate and thereby cause overproduction of lactate, even in the presence of adequate oxygen levels (16). In addition to allosteric regulation, $\mathrm{PDH}$ activity is controlled by $\mathrm{PDH}$ kinases (PDKs) that inhibit PDH enzyme activity by phosphorylation $(19,20)$ and PDH phosphatases that catalyze reciprocal dephosphorylation (18). Sirtuin 4 (SIRT4) was recently identified as a mitochondrial lipoamidase inhibiting PDH activity (21). PDK1-PDK4 are regulated at the transcriptional level via signaling cues involving factors such as AMP-dependent protein kinase (AMPK) (22), PPARs (23), and HIF1 (24). A possible role of impaired PDH function and abnormal AMPK activation in $\mathrm{ME} / \mathrm{CFS}$ has been proposed (25).

In summary, previous findings support that $\mathrm{PDH}$ dysregulation and changes in serum amino acids may be involved in the pathomechanism of ME/CFS. We hypothesized that ME/CFS is associated with defective oxidative metabolism involving $\mathrm{PDH}$, leading to increased utilization of ketogenic amino acids to fuel the TCA cycle. In order to address this hypothesis, we studied amino acid levels in serum samples from $200 \mathrm{ME} / \mathrm{CFS}$ patients fulfilling the Canadian consensus criteria (1) and 102 healthy controls. To investigate possible disease mechanisms, we measured mRNA expression of relevant genes in peripheral blood mononuclear cells (PBMCs) of ME/CFS patients and healthy controls. We exposed cultured muscle cells to ME/CFS patient serum to determine if systemic blood-borne substances could affect cellular energy metabolism. 


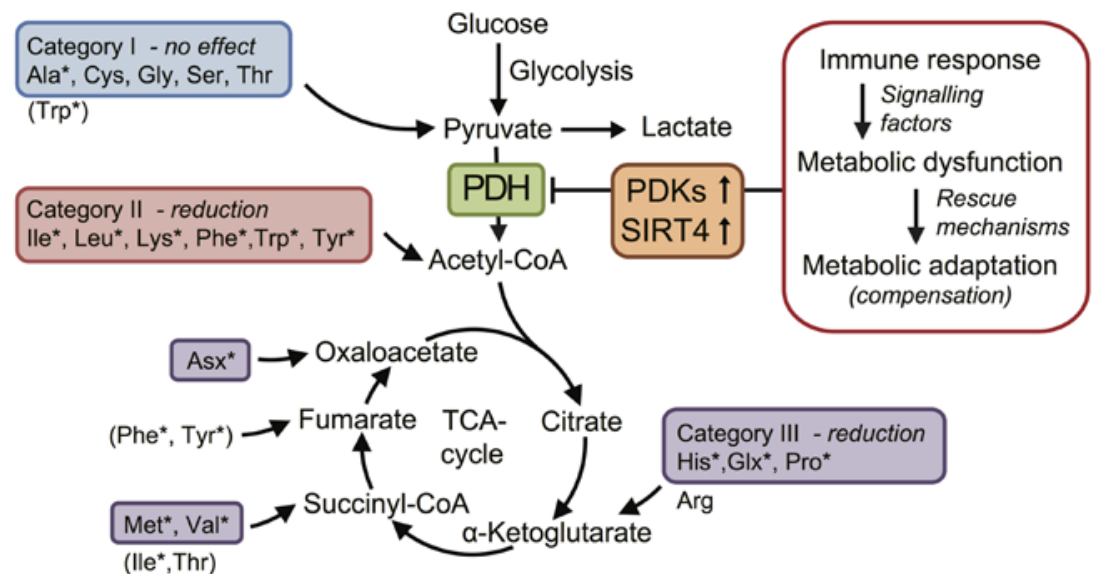

Figure 1. Hypothetic mechanism of ME/CFS linked to amino acid catabolism. According to this model, ME/CFS is caused by immune interference with an unidentified target, potentially a signaling factor, which ultimately causes metabolic dysfunction and induction of secondary rescue mechanisms. We hypothesize that aberrant PDK and SIRT4-mediated inhibition of $\mathrm{PDH}$, and consequent obstruction(s) in central energy metabolism, occurs early during ME/CFS pathogenesis, followed by metabolic adaptations serving to maintain ATP production. The result will be increased consumption of amino acids not depending on PDH to fuel oxidative metabolism via the TCA cycle. Such a mechanism would expectedly change the serum amino acid profile in patients, depending on the different entry stages of the amino acids into the catabolic pathway. Accordingly, for the purpose of serum amino acid profiling, the 20 standard amino acids were assigned into three categories: category I amino acids that may convert to pyruvate (i.e., dependent on PDH; Gly, Ser, Thr, Cys, Ala); category II amino acids that may metabolize to acetyl-CoA and fuel the TCA cycle (i.e., independent of PDH; Lys, Leu, Ile, Phe, Tyr, Trp); and category III amino acids that are anaplerotic and serve to replenish TCA cycle intermediates (i.e., independent of PDH; His, Pro, Met, Val, Glu + Gln = Glx, Asp + Asn = Asx). The asterisk indicates amino acids that were significantly reduced in serum of ME/CFS patients compared with healthy controls in this study (see Table 1 and 2).

\section{Results}

Serum concentrations of the 20 standard amino acids were assessed in $200 \mathrm{ME} / \mathrm{CFS}$ patients included in clinical trials led by Haukeland University Hospit al and 102 healthy controls. The initial analyses showed a distinct pattern when comparing amino acids with different entry points for oxidation via the mitochondrial pyruvate-TCA cycle axis (Figure 1). Therefore, amino acids were assigned to one of three categories, in agreement with the established paradigm for the major routes of amino acid catabolism (26):

Category I amino acids are converted to pyruvate, and therefore depend on $\mathrm{PDH}$ to be further oxidized. These are alanine (Ala), cysteine (Cys), glycine (Gly), serine (Ser), and threonine (Thr).

Category II amino acids enter the oxidation pathway as acetyl-CoA, which directly and independently of $\mathrm{PDH}$ fuels the TCA cycle for degradation to CO2. These are isoleucine (Ile), leucine (Leu), lysine (Lys), Phe, tryptophan (Trp), and tyrosine (Tyr).

Category III consists of anaplerotic amino acids that are converted to TCA cycle intermediates, thereby replenishing and supporting the metabolic capacity of the cycle. In this category, we included only amino acids that do not simultaneously belong to categories I or II. Arginine (Arg) was excluded from these analyses for reasons

described in Methods. This category consisted of methionine (Met) and valine (Val), which are converted to succinyl-CoA; histidine (His), Gln, Glu $(\mathrm{Gln}+\mathrm{Glu}=\mathrm{Glx})$, and proline (Pro), which are converted to $\alpha$-ketoglutarate; and asparagine (Asn) and aspartate (Asp), (Asn + Asp $=A s x)$, which are converted to fumarate or oxaloacetate.

This classification represents a simplified model. Several amino acids may have alternative degradation pathways with varying importance depending on the physiological context. The amino acids in categories I and III are solely glucogenic, whereas the amino acids in category II are ketogenic or both ketogenic and glucogenic. The serum concentration of Ala is highly influenced by its role in the glucose-Ala cycle, which serves to traffic amino groups from peripheral tissues to the liver via blood and was therefore analyzed separately.

Amino acids providing pyruvate as substrate for PDH (category I). There were no significant differences in mean serum levels of category I amino acids (Cys, Gly, Ser, Thr) between nonfasting ME/CFS patients and nonfasting healthy controls, except for Ala, for which there was a small but statistically significant reduction in patients $(P=0.027$, Cohen's $d=0.28$ ) (Table 1$)$. In stratified analyses, there were no differences for Gly and Cys in female ME/CFS patients compared with female healthy controls, but there were slight reductions in the mean concentrations of Ser and Thr $(P=0.010$, Cohen's $d=0.39$, and $P=0.010$, Cohen's $d=0.40$, respectively) (Table 1 ). In men, there were no differences in these amino acids between ME/CFS patients and healthy controls (Table 1). The sum of serum concentrations of these 4 category I amino acids (Ala excluded) is shown, with no differences in mean serum levels between ME/CFS patients and healthy controls (Figure 2A), nor in stratified analyses by sex (Figure 2B).

Amino acids fueling acetyl-CoA into TCA (category II). All the 6 amino acids in category II were significantly reduced in nonfasting ME/CFS patients compared with nonfasting healthy controls, with $P$ values of less than 0.001 for Ile, Leu, Phe, and Tyr and $P$ values of 0.001 and 0.009 for Lys and Trp, respectively (Table 1). The reductions in mean serum levels for all 6 amino acids were highly significant in women with ME/CFS compared with healthy women, with moderate effect sizes according to Cohen's $d$, varying from 0.35 to 0.75 . Comparing male ME/CFS patients to male healthy controls, 
Table 1. Serum levels of amino acids catabolized to pyruvate (category I), and to acetyl-CoA (category II), in nonfasting ME/CFS patients and healthy controls by sex

\begin{tabular}{|c|c|c|c|c|c|c|c|c|c|c|c|c|}
\hline & \multicolumn{4}{|c|}{ All patients and controls } & \multicolumn{4}{|c|}{ Women } & \multicolumn{4}{|c|}{ Men } \\
\hline & $\mathrm{ME}^{\mathrm{A}}$ & $H C^{B}$ & & & $\mathrm{ME}^{\mathrm{A}}$ & $H C^{B}$ & & & $M^{A}$ & $\mathrm{HC}^{\mathrm{B}}$ & & \\
\hline & $n=153$ & $n=102$ & $P$ value $^{\mathrm{c}}$ & $\begin{array}{c}\text { Effect } \\
\text { size }^{D}\end{array}$ & $n=124$ & $n=67$ & $P$ value $^{\mathrm{c}}$ & $\begin{array}{l}\text { Effect } \\
\text { size }^{D}\end{array}$ & $n=29$ & $n=35$ & $P$ value $^{c}$ & $\begin{array}{l}\text { Effect } \\
\text { size }^{D}\end{array}$ \\
\hline \multicolumn{13}{|c|}{$\begin{array}{l}\text { Category I (to Pyr) } \\
\text { ( } \mu \text { M, mean [SD]) }\end{array}$} \\
\hline Gly & $\begin{array}{l}290.7 \\
(79.8)\end{array}$ & $\begin{array}{l}273.3 \\
(76.3)\end{array}$ & 0.082 & 0.22 & $\begin{array}{l}295.4 \\
(85.6)\end{array}$ & $\begin{array}{l}285.5 \\
(83.5)\end{array}$ & 0.44 & & $\begin{array}{l}270.5 \\
(43.3)\end{array}$ & $\begin{array}{l}249.9 \\
(54.1)\end{array}$ & 0.096 & 0.42 \\
\hline Cys & $\begin{array}{l}259.0 \\
(26.0)\end{array}$ & $\begin{array}{l}258.9 \\
(26.9)\end{array}$ & 0.98 & & $\begin{array}{l}257.8 \\
(25.5)\end{array}$ & $\begin{array}{l}257.8 \\
(29.2)\end{array}$ & 0.99 & & $\begin{array}{l}264.0 \\
(28.1)\end{array}$ & $\begin{array}{l}261.0 \\
(22.1)\end{array}$ & 0.65 & \\
\hline Thr & $\begin{array}{l}135.9 \\
(32.7)\end{array}$ & $\begin{array}{l}143.2 \\
(34.4)\end{array}$ & 0.090 & 0.22 & $\begin{array}{l}134.5 \\
(33.8)\end{array}$ & $\begin{array}{l}149.2 \\
(38.8)\end{array}$ & 0.010 & 0.40 & $\begin{array}{l}141.7 \\
(26.8)\end{array}$ & $\begin{array}{l}131.8 \\
(19.8)\end{array}$ & 0.11 & \\
\hline \multicolumn{13}{|c|}{$\begin{array}{l}\text { Category II (to ac-CoA) } \\
\text { ( } \mu \mathrm{M}, \text { mean [SD]) }\end{array}$} \\
\hline Phe & $\begin{array}{l}68.2 \\
(13.1)\end{array}$ & $\begin{array}{l}76.6 \\
(11.8)\end{array}$ & $<0.001$ & 0.67 & $\begin{array}{c}67.5 \\
(12.5)\end{array}$ & $\begin{array}{l}77.0 \\
(12.7)\end{array}$ & $<0.001$ & 0.75 & $\begin{array}{l}71.4 \\
(15.1)\end{array}$ & $\begin{array}{c}75.8 \\
(10.0)\end{array}$ & 0.19 & \\
\hline Tyr & $\begin{array}{c}63.1 \\
(15.9)\end{array}$ & $\begin{array}{l}73.0 \\
(19.5)\end{array}$ & $<0.001$ & 0.56 & $\begin{array}{c}62.1 \\
(15.8)\end{array}$ & $\begin{array}{c}72.3 \\
(21.6)\end{array}$ & 0.001 & 0.62 & $\begin{array}{c}67.3 \\
(16.4)\end{array}$ & $\begin{array}{c}74.1 \\
(14.8)\end{array}$ & 0.086 & 0.44 \\
\hline Ile & $\begin{array}{c}70.3 \\
(20.0)\end{array}$ & $\begin{array}{l}80.7 \\
(23.4)\end{array}$ & $<0.001$ & 0.48 & $\begin{array}{c}67.0 \\
(17.3)\end{array}$ & $\begin{array}{c}77.9 \\
(26.3)\end{array}$ & 0.003 & 0.49 & $\begin{array}{c}84.4 \\
(24.8)\end{array}$ & $\begin{array}{l}86.0 \\
(15.4)\end{array}$ & 0.76 & \\
\hline $\operatorname{Trp}^{\mathrm{E}}$ & $\begin{array}{l}73.4 \\
(14.6)\end{array}$ & $\begin{array}{c}77.8 \\
(12.0)\end{array}$ & 0.009 & 0.28 & $\begin{array}{l}72.0 \\
(14.4)\end{array}$ & $\begin{array}{l}76.7 \\
(12.7)\end{array}$ & 0.022 & 0.35 & $\begin{array}{c}79.3 \\
(14.2)\end{array}$ & $\begin{array}{c}79.9 \\
(10.3)\end{array}$ & 0.85 & \\
\hline AlaF (category I) & $\begin{array}{l}423.5 \\
(93.5)\end{array}$ & $\begin{array}{l}447.9 \\
(81.0)\end{array}$ & 0.027 & 0.28 & $\begin{array}{l}420.3 \\
(91.0)\end{array}$ & $\begin{array}{l}449.9 \\
(87.2)\end{array}$ & 0.029 & 0.33 & $\begin{array}{c}437.1 \\
(104.1)\end{array}$ & $\begin{array}{l}444.1 \\
(68.7)\end{array}$ & 0.76 & \\
\hline
\end{tabular}

${ }^{A} \mathrm{ME}, \mathrm{ME} / \mathrm{CFS}$ patients, nonfasting. ${ }^{\mathrm{B}} \mathrm{HC}$, healthy controls, nonfasting. ${ }^{\mathrm{C}} P$ values from independent $t$ tests (equal variances not assumed). ${ }^{\mathrm{D}} \mathrm{Effect}$ sizes from Cohen's $d$ test. ETrp may also be catabolized to Pyr through Ala. ${ }^{F}$ Ala was excluded from category I in this analysis, since it is involved in the glucose-Ala cycle transporting amino groups from peripheral tissues to liver via blood. This cycle exerts major influence on the serum concentration of Ala, masking potential effects due to Ala oxidation. Pyr, pyruvate; ac-CoA, acetyl-CoA.

there were no significant differences in category II amino acids, with a trend for reduction in mean serum level of Tyr in patients ( $P=0.086$, Cohen's $d 0.44)$ (Table 1$)$. The sum of serum concentrations for the 6 category II amino acids was significantly lower in ME/CFS patients compared with healthy controls (Figure 2C) as well as in stratified analysis in women but not in men (Figure 2D). These effects were not caused by differences in statistical power due to higher number of women included compared with men (see Statistical analyses in the Methods).

Anaplerotic amino acids (category III). The mean serum levels of anaplerotic (category III) amino acids was significantly reduced in nonfasting ME/CFS patients relative to nonfasting healthy controls, with low to moderate effect sizes (Cohen's $d 0.28-0.60$ ) (Table 2). For reasons explained in the Supplemental Methods (supplemental material available online with this article; doi:10.1172/jci.insight.89376DS1), the concentrations of Gln and Glu (Glx) and of Asp and Asn (Asx) were presented as the sum for the respective pairs. Reductions in anaplerotic amino acids were observed in female ME/CFS patients, but not in men, compared with controls (Table 2). The sum of serum concentrations for the anaplerotic amino acids was reduced for the entire cohort of ME/CFS patients compared with controls (Figure 2E). In separate analyses, there was a significant reduction in the sum of serum concentrations of anaplerotic amino acids in female ME/CFS patients, but not in male patients (Figure $2 \mathrm{~F}$ ).

Comparison between nonfasting and fasting $M E / C F S$ patients. The comparisons of serum concentrations of all amino acids, between the overnight fasting and nonfasting ME/CFS patients, by sex are shown in Supplemental Tables 1 and 2. Serum concentrations were lower in overnight fasting patients for category II amino 

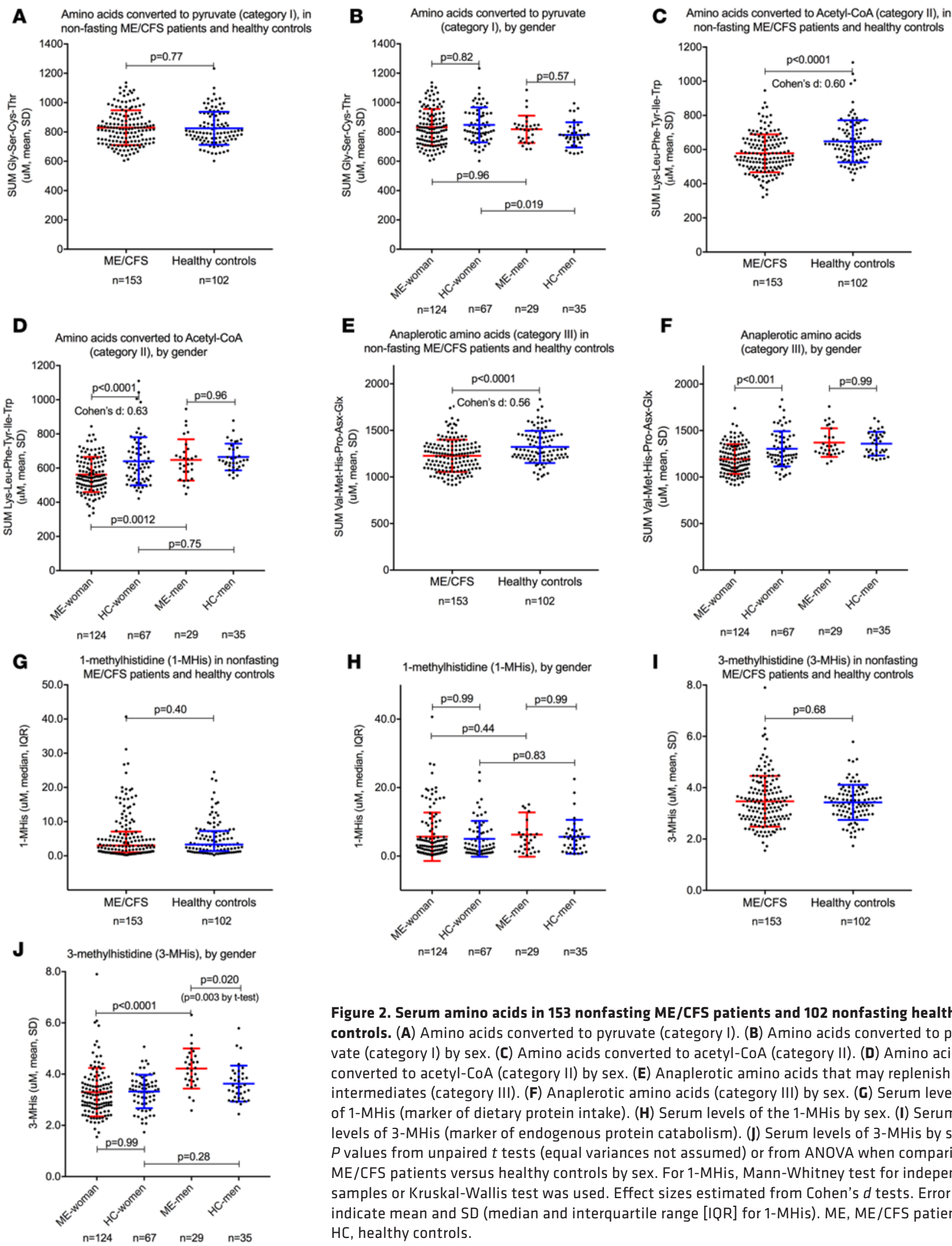

Figure 2. Serum amino acids in $\mathbf{1 5 3}$ nonfasting ME/CFS patients and 102 nonfasting healthy controls. (A) Amino acids converted to pyruvate (category I). (B) Amino acids converted to pyru vate (category I) by sex. (C) Amino acids converted to acetyl-CoA (category II). (D) Amino acids converted to acetyl-CoA (category II) by sex. (E) Anaplerotic amino acids that may replenish TCA intermediates (category III). (F) Anaplerotic amino acids (category III) by sex. (G) Serum levels of 1-MHis (marker of dietary protein intake). (H) Serum levels of the 1-MHis by sex. (I) Serum levels of 3-MHis (marker of endogenous protein catabolism). (J) Serum levels of 3-MHis by sex. $P$ values from unpaired $t$ tests (equal variances not assumed) or from ANOVA when comparing ME/CFS patients versus healthy controls by sex. For 1-MHis, Mann-Whitney test for independent samples or Kruskal-Wallis test was used. Effect sizes estimated from Cohen's $d$ tests. Error bars indicate mean and SD (median and interquartile range [IQR] for 1-MHis). ME, ME/CFS patients; $\mathrm{HC}$, healthy controls. 
Table 2. Serum levels of anaplerotic amino acids (category III), metabolites that may affect endothelial function, and markers of protein catabolism in nonfasting ME/CFS patients and healthy controls by sex

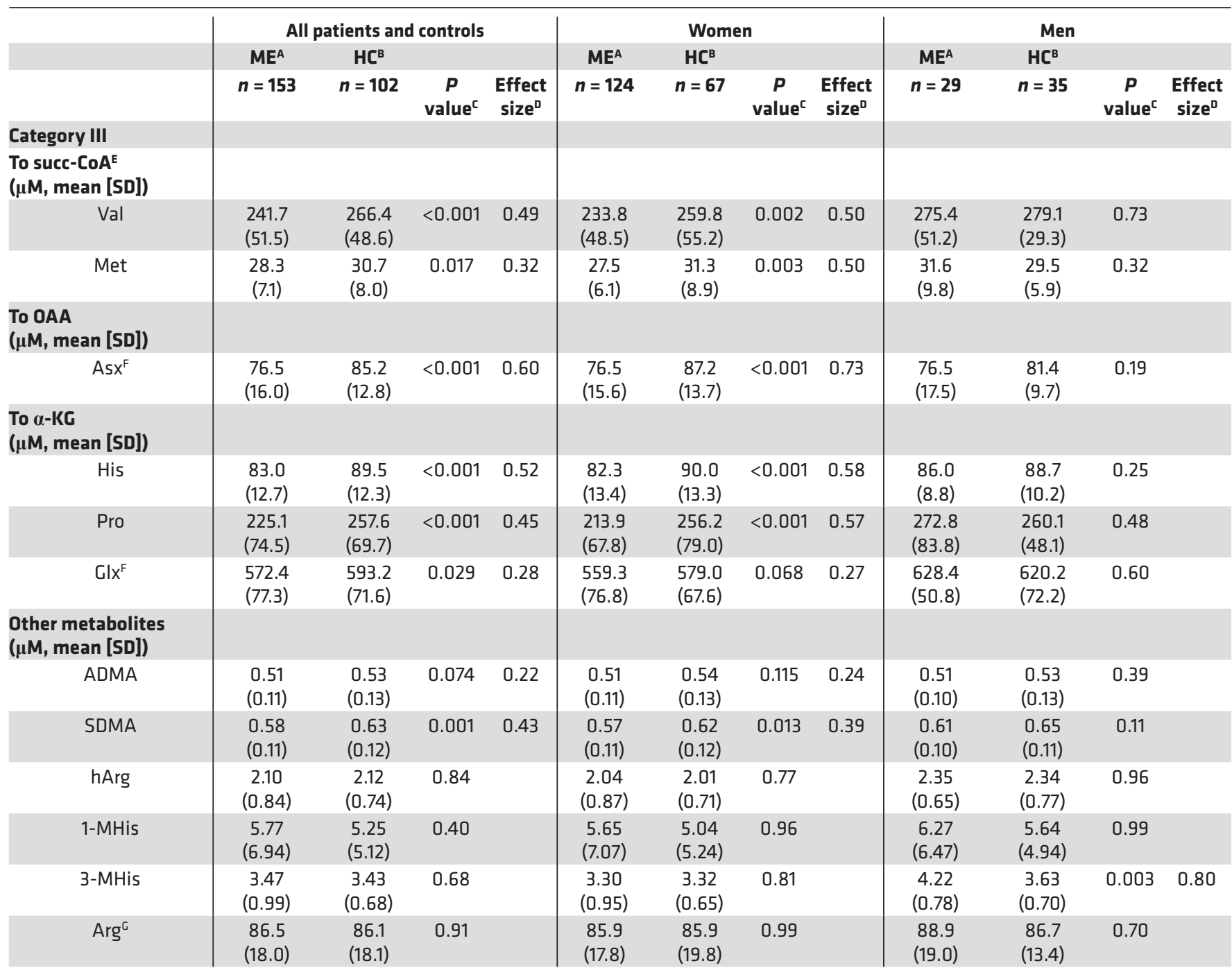

${ }^{A} M E, M E / C F S$ patients, nonfasting. ${ }^{B} \mathrm{HC}$, healthy controls, nonfasting. ${ }^{\mathrm{C}} P$ values from independent $t$ tests (equal variances not assumed), except for 1-MHis analyzed by Mann-Whitney test. ${ }^{D}$ Effect sizes from Cohen's $d$ test. Elle and Thr may also replenish the TCA cycle at succ-CoA level. ${ }^{\text {ED }}$ uring storage, Gln converts partly to Glu and Asn converts partly to Asp. The respective sums of these two amino acid pairs were calculated: Gln + Glu = Glx and Asp + Asn = Asx. ' Initial analyses demonstrated significantly higher mean serum levels of Arg in healthy controls sampled in 2012 versus those sampled in 2015, similar to ME/CFS patient samples from the KTS2 study (sampled in 2010) versus serum samples harvested from ME/CFS patients in 2015. These data were in agreement with in-house storage data from Bevital (http://www.bevital.no). Thus, analyses for Arg were performed excluding samples from 2010 and 2012, with remaining samples harvested during late 2014 and 2015 from 134 nonfasting ME/CFS patients (108 women and 26 men) and 40 nonfasting healthy controls (29 women and 11 men). succ-CoA, succinyl-CoA; OAA, oxaloacetate; $\alpha$-KG, $\alpha$-ketoglutarate.

acids Ile, Phe, Trp, and Tyr, in addition to Ala. There were trends for lower mean serum levels of Pro, Met, and Arg in serum of overnight fasting patients but no differences for the other amino acids or metabolites.

Other modified amino acids. Based on a study that showed endothelial dysfunction in ME/CFS patients (27), we measured serum concentrations of amino acids and derivatives known to affect endothelial function. Arg, asymmetric dimethylarginine (ADMA), and homoarginine (hArg) showed similar serum levels between ME/CFS patients and controls both in women and men (Table 2). For symmetric dimethylarginine (SDMA), there was a significantly reduced mean serum level in women with ME/ CFS, but not in men, compared with healthy controls. 1-methylhistidine (1-MHis), a marker of dietary protein intake from mainly animal and meat sources (28), showed no differences between ME/CFS 
patients and controls, neither in women nor in men (Table 2 and Figure 2, G and H). Concentrations of 3-methylhistidine (3-MHis), a marker of endogenous protein catabolism (e.g., muscle atrophy) (28, $29)$, were significantly higher in men with ME/CFS compared with healthy men $(P=0.003$ by $t$ test, Cohen's $d=0.80$ ), while there was no corresponding difference in 3-MHis between female ME/CFS patients and healthy women (Table 2 and Figure 2, I and J).

Amino acids and their relationship to other ME/CFS patient characteristics. Among nonfasting women with $\mathrm{ME} / \mathrm{CFS}$, a correlation matrix demonstrated highly significant bivariate correlations for all combinations of the category II amino acids (Ile, Leu, Lys, Phe, Trp, Tyr) $(P<0.001$, with Pearson's $r$ varying from 0.44 to 0.93 (Supplemental Table 3). Among the male ME/CFS patients, similar patterns of correlations were seen (Supplemental Table 3). In contrast, fewer and weaker correlations were found between category I and category II amino acids. However, for Thr there were several marked correlations to category II amino acids, possibly reflecting that Thr has alternative oxidation routes that are linked to the TCA cycle without being converted to pyruvate. Furthermore, there were highly significant correlations for all combinations of category II and category III amino acids but fewer significant correlations between category I and category III amino acids (Supplemental Table 4).

Sex appeared to be an important factor in interpretation of the results, with significant reductions of mean serum levels of category II and III amino acids evident in female ME/CFS patients. To evaluate the possibility that observed changes in amino acid patterns could be caused by other confounding factors, we performed correlation analyses and ANOVA to compare amino acid levels in serum and clinical variables (age, BMI, level of physical activity, ME/CFS severity, and ME/CFS disease duration). Increasing age correlated significantly with increasing levels of Cys, Gly, Ile, Leu, Lys, and Tyr among women with ME/ CFS but only with Cys among men with ME/CFS (Supplemental Table 3). There were no significant correlations between serum levels of amino acids and physical activity, assessed as number of steps registered electronically per 24 hours in the ME/CFS group, except for a significant negative correlation with Cys in women (Supplemental Table 3). Among women with ME/CFS, there were significant positive correlations between BMI and Cys, Ile, Leu, Phe, and Tyr and significant negative correlations between BMI and Ser and Thr (Supplemental Table 3). To exclude the possibility that differences in BMI could account for the observed differences in serum amino acid levels in women with ME/CFS, the patients were assigned to four BMI groups, ranging from underweight to obese (Supplemental Tables 5 and 6). When comparing these groups, there were significant associations between higher BMI and higher serum levels of the category II amino acids Leu, Phe, Tyr, and Ile. However, among female ME/CFS patients, in all BMI groups including obese, the mean serum levels of these amino acids were lower than in healthy women (Supplemental Table 5). In men with ME/CFS, no significant associations between BMI groups and serum amino acids belonging to category I or II were seen, except for a significant positive association between Cys with BMI (Supplemental Table 5). The corresponding analyses comparing BMI groups and amino acids from category III and for modified amino acids are shown in Supplemental Table 6.

Further, the patients were also assigned to groups of disease severity, disease duration, physical activity level, and quality of life. Serum levels of category I or category II amino acids were not associated with ME/CFS disease severity (Supplemental Table 7). A significant positive association between ME/CFS disease duration and serum amino acid level was found only for Phe in female patients (Supplemental Table 8). There were no significant associations between mean serum amino acid levels (category I and II) and physical activity, except for a negative association of serum Cys in female ME/CFS patients (Supplemental Table 9). There were significant associations between higher serum ADMA levels and higher physical activity $(P=0.036)$ and between higher serum level of the marker of dietary protein intake 1-MHis and higher level of physical activity $(P=0.024)$ in female ME/CFS patients, with a trend in male patients $(P=0.086)$ (Supplemental Table 10). The associations between quality of life assessed by "SF36mean5" and amino acids (categories I and II) are shown in Supplemental Table 11, with a significant association between a higher "SF36mean5" score and a higher mean serum level of the category I amino acid Ser ( $P$ $=0.023)$ and with trends for Gly $(P=0.056)$ and Thr $(P=0.065)$, but only in female ME/CFS patients.

$P D H$-related gene expression ( $m R N A)$ in PBMCs. To investigate whether the observed effects on the serum amino acid profile in ME/CFS patients could be explained by changes in PDH function, we compared mRNA levels of PDH-related genes in PBMCs from nonfasting ME/CFS patients and nonfasting healthy controls (Figure 3). We found significantly increased mRNA expression in ME/CFS patients of the inhibitory kinases $P D K 1(P=0.002), P D K 2(P=0.022)$, and $P D K 4(P=0.006)$, whereas $P D K 3$ was unchanged 
A
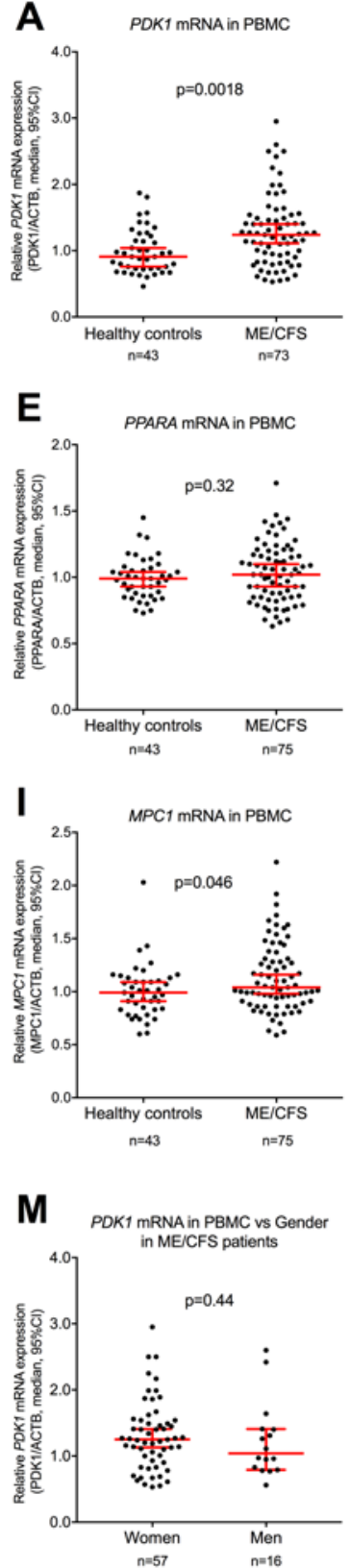
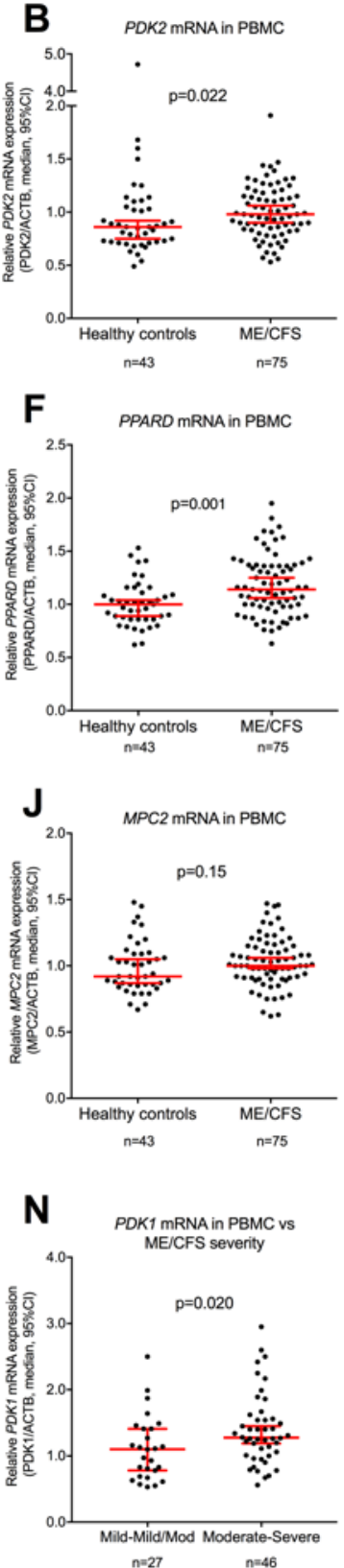
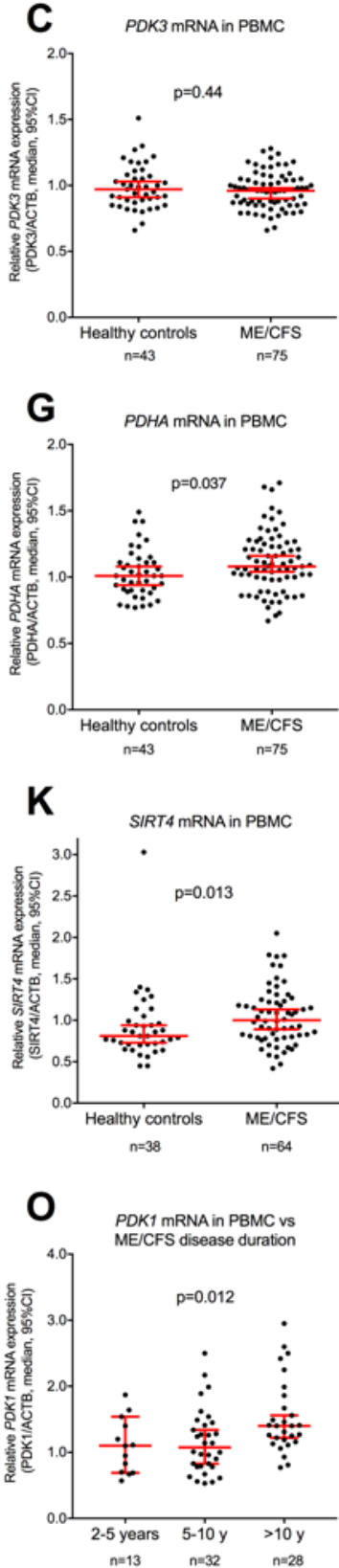
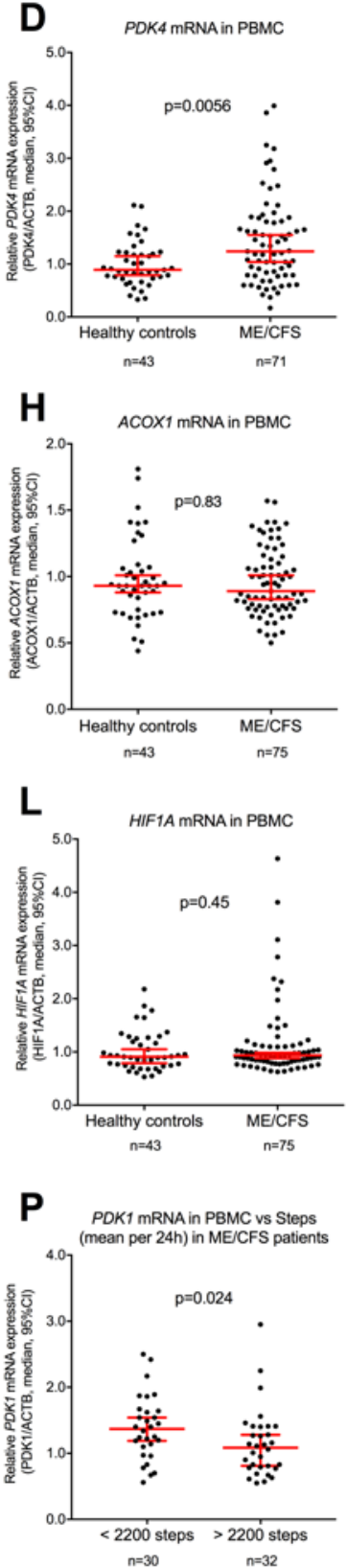

Figure 3. Quantitative RT-PCR for mRNA expression levels in peripheral blood mononuclear cells (PBMCs) of ME/CFS patients and healthy controls. mRNA expression levels in PBMCs from 75 nonfasting ME/CFS patients and 43 nonfasting healthy controls, normalized according to coamplified internal $\beta$-actin $(A C T B$ ) in duplex qRT-PCR and calculated relative to the mean of healthy controls. (A) Pyruvate dehydrogenase kinase 1 (PDK1) mRNA in PBMCs from ME/CFS patients and healthy controls. Similar analyses are shown for (B) PDK2, (C) PDK3, (D) PDK4, (E) PPARA $\alpha(P P A R A),($ F) PPAR $\delta$ (PPARD), (G) pyruvate dehydrogenase E1, subunit $\alpha$ (PDHA), (H) acyl-coenzyme A oxidase 1 (ACOX1), (I) mitochondrial pyruvate carrier 1 (MPC1), (J) MPC2, (K) sirtuin 4 (SIRT4), (L) HIF-1 $\alpha$ (HIF1A), (M) PDK1 mRNA in PBMCs of ME/CFS patients versus sex, (N) PDK1 mRNA in PBMCs versus ME/CFS severity, (0) PDK1 mRNA in PBMCs versus ME/CFS duration, and (P) PDK1 mRNA in PBMCs versus steps (mean) per 24 hours in ME/CFS patients. $P$ values were from Mann-Whitney $U$ test for independent samples (A-N and $\mathbf{P}$ ) and from Kruskal-Wallis test (0). Error bars indicate median with $95 \% \mathrm{CI}$. All samples in a qRT-PCR assay were run in triplicate on the same plate. Of 75 samples from patients and 43 from healthy controls, two samples for PDK1 and four samples for PDK4 were excluded due to unsuccessful amplification. For SIRT4, due to a low expression level, 11 samples from ME/CFS patients and 5 samples from healthy controls were excluded due to high SD ( $\geq 30 \%$ ) among triplicates (see the Methods). Sensewear bracelet data for physical activity for 7 consecutive days were available from 62 of the 75 patients.

(Figure 3, A-D). Also, the mitochondrial lipoamidase and PDH inhibitor SIRT4 was significantly upregulated in ME/CFS patients $(P=0.013)$ (Figure $3 \mathrm{~K})$. Among the PPAR transcription factors, PPAR $\delta$ $(P P A R D)$ was upregulated in PBMCs of ME/CFS patients $(P=0.001)$ (Figure $3 \mathrm{~F})$. There were no differences for PPAR $\alpha$ (PPARA) (Figure 3E), the PPAR transcriptional target and peroxisomal fatty acid $\beta$-oxi- 
A Correlation of PDK1 vs PPARD mRNA

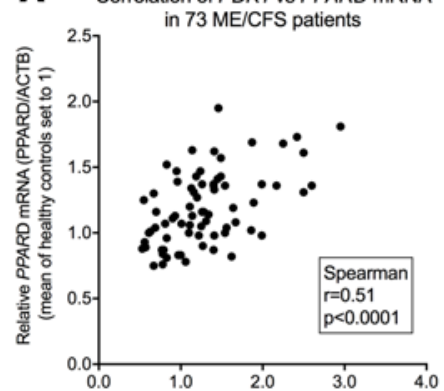

Relative PDK1 mRNA (PDK1/ACTB (mean of healthy controls set to 1 )

E Correlation of PDK4 vs SIRT4 mRNA

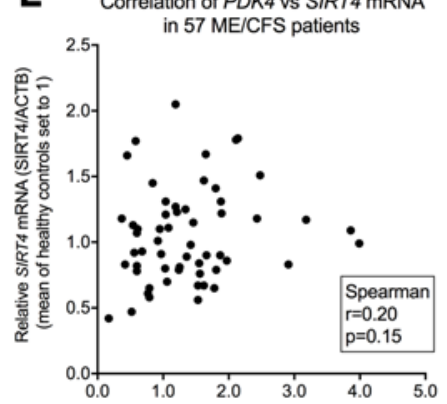

Relative PDK1 mRNA (PDK1/ACTB) (mean of healthy controls set to 1 )

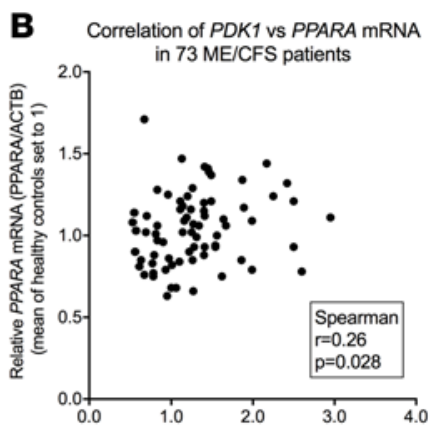

Relative PDK1 mRNA (PDK1/ACTB) (mean of healthy controls set to 1 )

F Correlation of PPARD vs SIRT4 mRNA

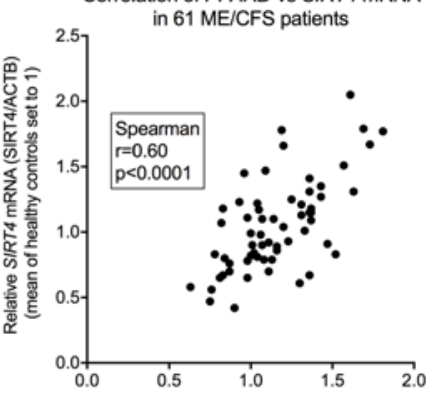

Relative PDK1 mRNA (PDK1/ACTB) (mean of healthy controls set to 1 )

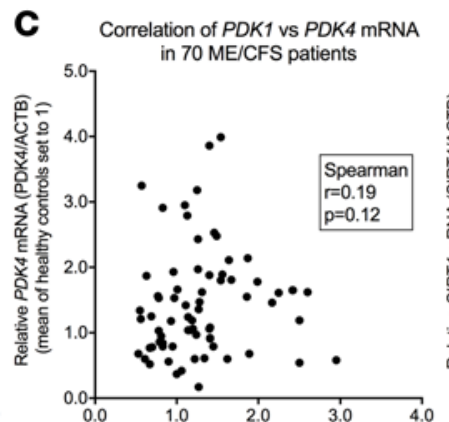

Relative PDK1 mRNA (PDK1/ACTB) (mean of healthy controls set to 1 )

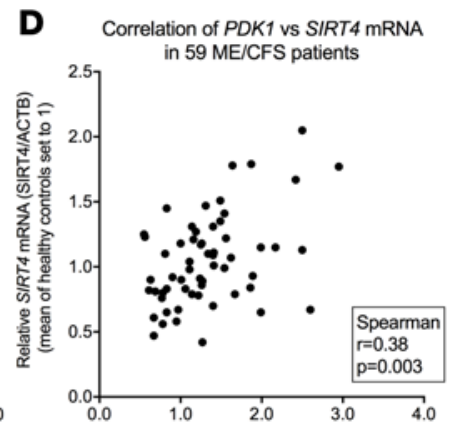

Relative PDK1 mRNA (PDK1/ACTB) (mean of healthy controls set to 1)

Figure 4. Correlation analyses between mRNA expression levels in peripheral blood mononuclear cells (PBMCs) of ME/CFS patients. mRNA expression levels in PBMCs from nonfasting ME/CFS patients, normalized according to coamplified internal $\beta$-actin (ACTB) in duplex qRT-PCR and calculated relative to the mean of healthy controls. (A) Correlation of pyruvate dehydrogenase kinase 1 (PDK1) and PPAR $\delta$ (PPARD) mRNA levels in PBMCs of ME/CFS patients. (B) Correlation of PDK1 and PPAR $\alpha$ (PPARA). (C) Correlation of PDK1 and PDK4. (D) Correlation of PDK1 and sirtuin 4 (SIRT4). (E) Correlation of PDK4 and SIRT4. (F) Correlation of PPARD and SIRT4. (C) Correlation of PPARA and SIRT4. (H) Correlation of PPARD and PPARA. P values from Spearman correlation analyses. Of 75 samples from patients, two samples for $P D K 1$ and four samples for PDK4 were excluded due to unsuccessful amplification. For SIRT4, due to a low expression level 11 samples from ME/CFS patients were excluded, due to high SD ( $\geq 30 \%)$ among triplicates (see the Methods). In three samples with SIRT4 mRNA data, PDK and PPAR mRNAs were not analyzed, leaving 61 samples for SIRT4 correlation analyses.

dation enzyme, acyl-coenzyme A oxidase 1 (ACOX1), or the transcription factor HIF1 $\alpha$ (HIF1A) (Figure 3, $\mathrm{H}$ and $\mathrm{L})$. Pyruvate dehydrogenase $\mathrm{E} 1 \alpha(P D H A)$ mRNA was slightly upregulated in ME/CFS patients $(P$ $=0.037$ ) (Figure 3G). PPAR $\gamma(P P A R G)$ mRNA expression in PBMCs was below the detection limit of the analysis (data not shown). Analyses of the mitochondrial pyruvate carriers (MPCs) revealed a borderline significant upregulation of MPC1 in ME/CFS patients compared with healthy controls $(P=0.046)$ but no difference for $M P C 2$ (Figure 3, I and J).

While there were sex-specific differences in mean serum levels of amino acids in ME/CFS patients, the increased mRNA levels in PBMCs from ME/CFS patients versus healthy controls were similar in men and women for PDK1 (Figure 3M), PDK4, PPARA, PPARD, and SIRT4 (Supplemental Figure 1, A, E, I, and M).

For $P D K 1$ gene expression, there were significant associations with ME/CFS disease severity (higher $P D K 1$ mRNA level in moderate/severe versus mild/mild-moderate groups), with ME/CFS disease duration (higher $P D K 1$ mRNA level with increasing duration), and with physical activity level assessed as the mean steps per 24 hours (higher $P D K 1$ mRNA level with lower activity) (Figure 3, N-P). The corresponding associations were not found for PDK4 mRNA (Supplemental Figure 1, B-D), for PPARA (Supplemental Figure 1, F-H), for PPARD (Supplemental Figure 1, J-L), or for SIRT4 (Supplemental Figure 1, N-P).

The mRNA expression level of $P D K 1$ correlated highly with $P P A R D(r=0.51, P<0.0001)$ (Figure 4A), with SIRT4 ( $r=0.38, P=0.003$ ) (Figure 4D), and weakly, but significantly, with PPARA $(r=0.26, P$ $=0.028)$ (Figure 4B). There was no significant correlation between $P D K 1$ and $P D K 4(r=0.19, P=0.12)$ (Figure 4C). Further, there were highly significant correlations between SIRT4 and PPARD $(r=0.60, P<$ $0.0001)$ and SIRT4 and PPARA $(r=0.52, P<0.0001)$ but no significant correlation between SIRT4 and $P D K 4(r=0.20, P=0.15)$ (Figure 4, E-G). These data were substantiated by a highly significant correlation between PPARA and PPARD mRNA levels $(r=0.75, P<0.0001)$ (Figure $4 \mathrm{H})$. For PDK1, PDK4, PPARA, or 
A

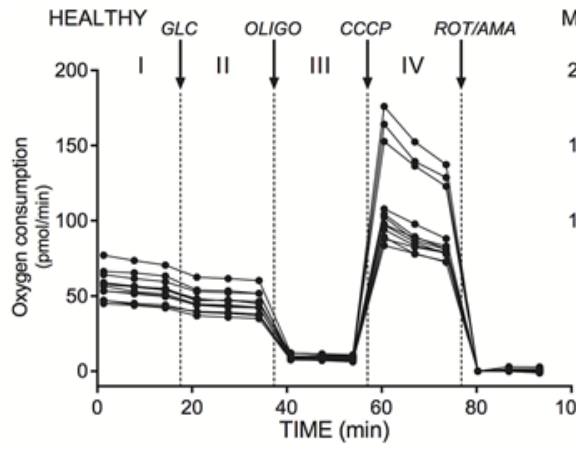

C
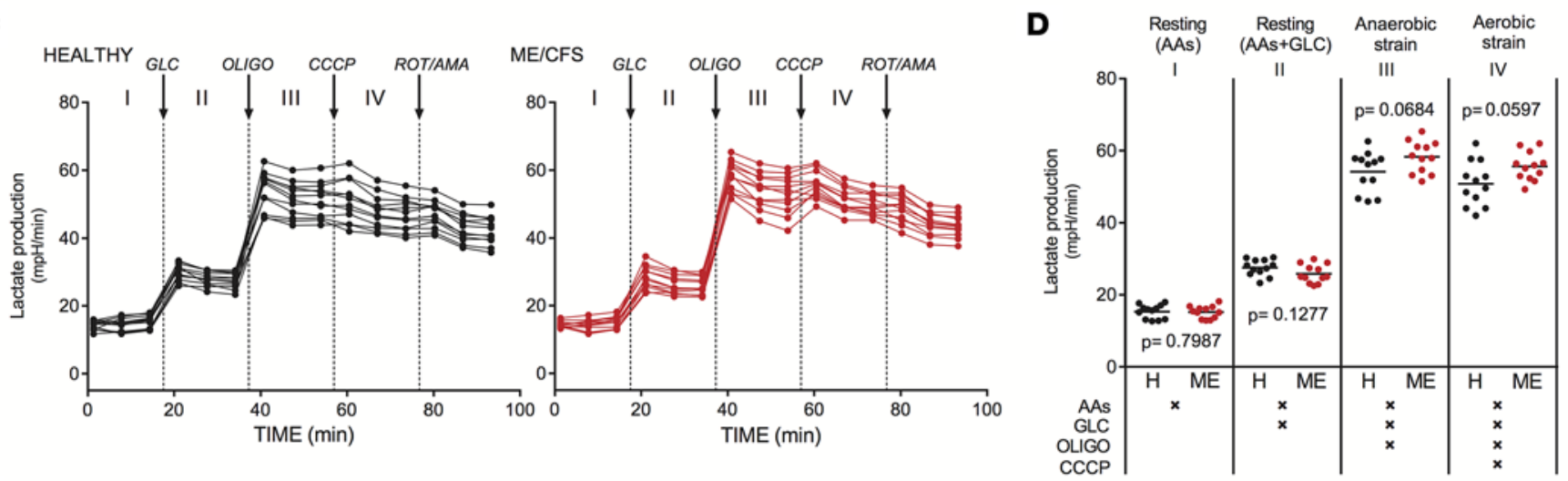

E

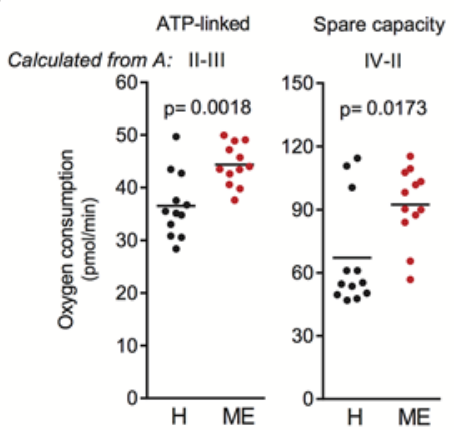

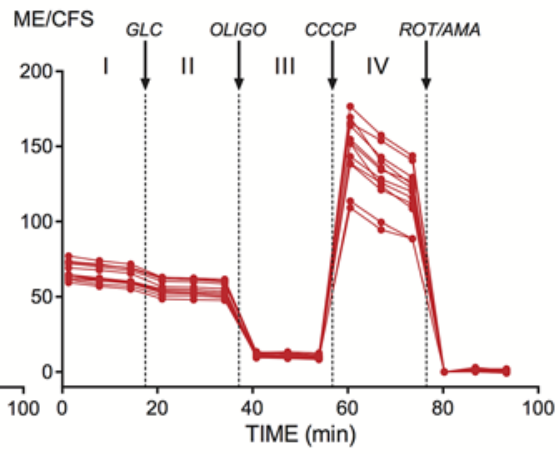

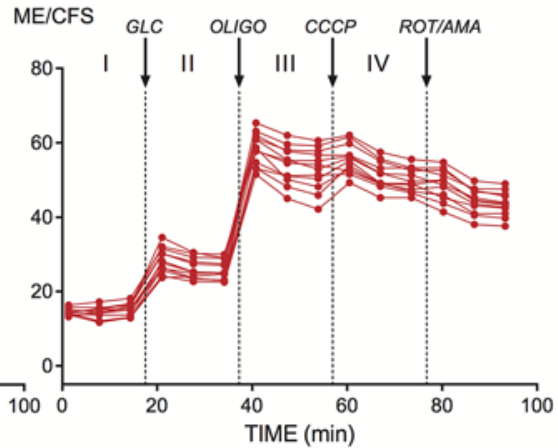

D

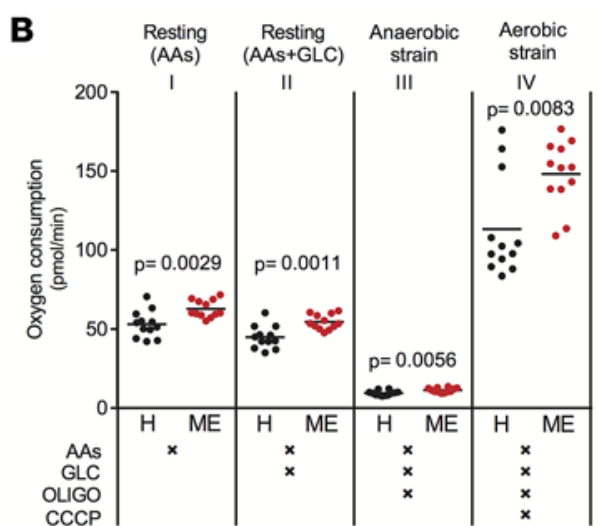

$\mathbf{F}$

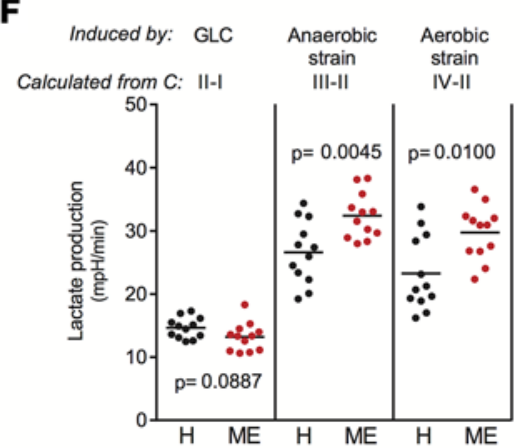

Figure 5. Effects of ME/CFS patient and healthy control serum on muscle cell metabolism. Rates of oxygen consumption and lactate production were measured simultaneously in cultures of human muscle cells (HSMM) after exposure (6 days) to serum from healthy individuals ( $n=12$ ) or ME/CFS patients $(n=12)$. Glucose (GLC), oligomycin (OLICO), carbonyl cyanide 3-chlorophenylhydrazone (CCCP), and rotenone/antimycin A (ROT/AMA) were administered sequentially during the analysis to assess specific properties of cellular energy metabolism. The consequent energetic states of the cells were classified as follows: I Resting (AAs), II Resting (AAs+GLC), III Anaerobic strain, and IV Aerobic strain, as described in the Methods. (A) Recordings of oxygen consumption rate are shown for muscle cells preexposed to healthy control (black) and ME/CFS patient (red) serum. The substance additions and the resultant energetic conditions of the cells are indicated (conditions I-IV). (B) Statistical analysis of the data in A. (C) Recordings of the lactate production rate from the same experiment as A and B. (D) Statistical analysis of the data in C. (E) Specific descriptors of mitochondrial respiration were calculated as indicated, based on the data in $\mathbf{A}$. (F) Specific descriptors of inducible lactate production were calculated as indicated, based on the data in $\mathbf{C}$. The analysis was performed with 5 replicate wells for each serum sample and is representative of 3 separate experiments. Statistical comparisons between healthy controls and ME/CFS samples were performed by Mann-Whitney $U$ test for independent samples.

PPARD mRNA expressions, there were no significant correlations to age or to BMI (Supplemental Figure $1, \mathrm{Q}-\mathrm{X})$. Among the 75 nonfasting ME/CFS patients analyzed for mRNA expression in PBMCs, there were no significant correlations between serum levels of category I or II amino acids and mRNA expression levels in PBMCs for PDKs, SIRT4, PPARA, or PPARD (data not shown).

Effects of ME/CFS patient serum on mitochondrial respiration and lactate production in cultured skeletal muscle cells. In order to explain the hallmarks of ME/CFS symptoms, i.e., postexertional malaise and 
poor recovery, one would expect relevant metabolic defects to be operative in skeletal muscle cells. We were not able to measure energy metabolism directly in muscle tissue of our patients. However, to study the influence of possible blood-borne substances in ME/CFS pathophysiology, we investigated energy metabolism in cultured human skeletal muscle cells (HSMM) exposed to serum from $12 \mathrm{ME} / \mathrm{CFS}$ patients (including 3 patients with very severe disease and 6 patients with severe disease) and 12 healthy controls. The study was designed to assess mitochondrial respiration and lactate production by measurement of oxygen consumption rate (OCR) and extracellular acidification rate (ECAR), respectively, in presence of amino acids and glucose as substrates. For didactic purposes, the conditions mirroring different energetic states of the cells were defined as conditions I-IV, as explained in Methods.

Basal (resting) amino acid-driven mitochondrial respiration (condition I) was moderately increased in muscle cells exposed to ME/CFS serum for 6 days (Figure 5, A and B), and this effect was also present when glucose was added (condition II). Subsequent addition of the ATP synthase inhibitor oligomycin (condition III) demonstrated that nearly all respiratory activity was linked to ATP production, confirming that the integrity of the oxidative phosphorylation system was intact in cells cultured in the presence of ME/CFS serum. There was, however, a minor increase in the remaining OCR (i.e., leak activity). Next, administration of the uncoupler carbonyl cyanide 3-chlorophenylhydrazone (CCCP) revealed a significantly increased respiratory capacity in cells exposed to ME/CFS serum (condition IV). The data also indicated that ATP-linked respiration (difference condition II-III) and spare respiratory capacity (difference condition IV-II) were increased after exposure to ME/CFS serum (Figure 5E).

The basal glycolytic rate (condition II) was similar in cells exposed to ME/CFS and control serum (Figure 5, C and D). However, there was a trend toward reduced glucose-induced rate in the cells cultured with ME/CFS serum (difference condition II-I) (Figure 5F). In contrast, the maximum glycolytic rate in presence of oligomycin tended to be slightly increased in cells exposed to ME/CFS serum (condition III), and this trend was also present after injection of CCCP (Figure 5, C and D). Further analysis of these data revealed that the lactate production caused by oligomycin (difference condition III-II), and by CCCP (difference condition IV-II), were significantly increased in cells exposed to ME/CFS compared with control serum (Figure 5F). Therefore, the cells exposed to ME/CFS serum displayed a metabolic change involving amplified lactate production under conditions of energetic strain.

In summary, serum from ME/CFS patients with severe disease was found to increase rates of mitochondrial oxidative metabolism and respiration in muscle cells, particularly under conditions of energetic strain. Additional experiments with shorter exposure showed that the effect of ME/CFS serum on mitochondrial respiration gradually increased depending on exposure time (data not shown).

\section{Discussion}

This study found that the serum amino acid profile was changed in a large and well-characterized ME/ CFS patient cohort, in a direction that suggests impaired mitochondrial pyruvate oxidation. This finding, in combination with increased mRNA expression of PDK1, PDK2, and PDK4, and of SIRT4, in PBMCs from patients, points to abnormalities in $\mathrm{PDH}$ regulation as a potential key factor in ME/CFS pathogenesis. Exposing cultured muscle cells to serum from ME/CFS patients indicated the presence of blood-borne substances affecting energy metabolism.

Analysis of serum from $200 \mathrm{ME} / \mathrm{CFS}$ patients and 102 healthy controls revealed that the concentrations of amino acids that enter mitochondrial oxidative metabolism at the level of acetyl-CoA (category II) were significantly reduced in female ME/CFS patients. In contrast, there were no major changes in amino acids that are converted to pyruvate (category I). Accordingly, ME/CFS appears to cause a specific reduction of energetic substrates that enter oxidation downstream of $\mathrm{PDH}$. This suggests that pyruvate catabolism is obstructed in ME/CFS patients, leading to increased utilization of acetyl-CoA-producing amino acids as alternative substrates fueling aerobic metabolism via the TCA cycle. The anaplerotic amino acids, which serve to maintain TCA cycle capacity (category III), were reduced in female ME/CFS patients. Our findings are in agreement with previous reports showing reductions of Gln, Glu, and Phe in smaller ME/CFS patient cohorts $(4,14)$. Interestingly, a study of ME/CFS patients found changes in urine amino acid concentrations consistent with the specific amino acid pattern we found in serum, and a reduced urinary excretion of the TCA cycle intermediates succinic acid and citric acid was also reported (15). A recent metabolomics study reported reduced concentrations of TCA cycle intermediates, accompanied by an increased pyruvate/isocitrate ratio, in ME/CFS plasma (30). This further supports the 
hypothesis that fueling of the TCA cycle by pyruvate is obstructed in ME/CFS patients, which is in line with the suggested PDH inhibition.

We found significant mRNA upregulation of $P D K 1, P D K 2$, and $P D K 4$ in PBMCs from ME/CFS patients. The expression of $P D K 1 \mathrm{mRNA}$ was associated with clinical characteristics such as longer disease duration, more severe ME/CFS, and reduced physical activity. The function of the PDKs is to inhibit PDH by phosphorylation under conditions in which pyruvate oxidation is to be repressed, such as in starvation $(19,20)$. An aberrant increase in PDK expression may therefore cause impaired PDH function in ME/ CFS, possibly via activity of PPAR transcription factors (23). PPARD mRNA was significantly upregulated in PBMCs of ME/CFS patients, while PPARA showed no difference compared with healthy controls. There was, however, a positive correlation between the expression of $P D K 1$ and both of these PPARs. Even if expression of the PPAR target gene ACOX1 was similar in ME/CFS patients and controls, it cannot be excluded that the PPARs contribute in the regulation of PDKs at some stage of ME/CFS disease development (23). The mitochondrial enzyme SIRT4 was recently reported to inhibit PDH activity by hydrolyzing lipoamide cofactors from the E2 component of the PDH complex (21). We found that SIRT4 mRNA expression was upregulated in PBMCs from ME/CFS patients and correlated significantly with mRNA expression of PDK1, PPARA, and PPARD. This suggests that $\mathrm{PDH}$ activity is inhibited by coordinated regulatory mechanisms in these patients. There was a small but significant increase in PBMC mRNA expression of $M P C 1$ but not of $M P C 2$. These MPCs were recently identified to play important roles in mitochondrial oxidative metabolism (31). The slight upregulation of MPC1 and of PDH-E1 $\alpha(P D H A)$ mRNAs might reflect compensatory mechanisms to counteract pyruvate accumulation due to reduced PDH activity.

The oxidation pathways of pyruvate and amino acids are tightly associated with the fatty acid oxidation machinery, converging at the level of acetyl-CoA in the mitochondrial compartment. An adaptive response to a low-energy state caused by a limitation in acetyl-CoA supply would be expected to involve multiple signaling factors serving to rescue energy homeostasis. Such signaling factors include AMPK, PPARs, and HIF1, which mediate context-dependent regulation of many metabolic pathways (22-24). Gaining more knowledge about the relative contributions of specific catabolic pathways in ME/CFS pathogenesis will be important to facilitate development of biomarkers and treatments for the disease.

The PDH inhibition in ME/CFS suggested in the present study, with compensatory use of amino acids as substrates for TCA oxidation, would also be expected to affect mitochondrial fatty acid $\beta$-oxidation in attempts to rescue energy homeostasis. A recent study assessed 612 metabolites in $45 \mathrm{ME} / \mathrm{CFS}$ patients and 39 healthy controls and reported multiple abnormalities in several pathways involved in lipid metabolism and mitochondrial energy metabolism (32). The most prominent changes in ME/CFS patients were widespread decreases in sphingolipids, glycosphingolipids, and phospholipids. These findings were consistent with a lower ATP and GTP turnover and with decreased amounts of branched amino acid metabolic intermediates. However, there were also indications of reduced mitochondrial fatty acid oxidation only in female ME/CFS patients (32). Adding to PDH inhibition, reduced mitochondrial fatty acid oxidation would expectedly compromise the supply of acetyl-CoA further and thereby increase the dependency of category II amino acids for alternative fueling of the TCA cycle, as shown in our study.

Sex-specific differences regarding changes in serum amino acid patterns were evident in our ME/CFS patients. While all 6 amino acids that may convert to acetyl-CoA were significantly reduced in female ME/ CFS patients compared with healthy women, male ME/CFS patients showed slight and not significant reductions in serum Tyr and Phe. Due to the relatively low number of male ME/CFS patients and male healthy controls included in the study, the statistical power to detect a difference among men was lower. However, the effect sizes were larger among women. In addition, from the 124 nonfasting female ME/CFS patients and 67 healthy women, separate analyses were performed in two nonoverlapping random groups of 33 female ME/ CFS patients and 33 healthy women, showing the same patterns, with a significant reduction in sum of serum concentrations of both category II and category III amino acids among women with ME/CFS. This argues for a differential and sex-specific use of serum-free amino acids in female ME/CFS patients. A previous study reported significantly increased urinary excretion of 3-MHis, which is a marker of endogenous protein catabolism, in a group of $100 \mathrm{ME} / \mathrm{CFS}$ patients, among which 73 were female (15). However, when we analyzed serum 3-MHis by sex, we found increased concentration only in male ME/CFS patients. This suggests that men, more than women, may utilize endogenous protein catabolism to provide substrates for the TCA cycle. Sex-specific differences in metabolism have been demonstrated, and may be operative in ME/CFS patients, involving fatty acids, amino acids, oxidative phosphorylation, and steroid metabolism (33). In contrast to the 
sex-specific effects on serum amino acids, the increased expression of PDKs and PPARD (mRNA in PBMCs) was similar in women and men with ME/CFS. Together, this indicates that the disease-causing mechanism triggers different compensatory effects in women and men. Thus, a common effector system for symptom maintenance may involve the PDK-PDH-lactate axis, with partly sex-specific compensatory (secondary) effects related to adaptations in energy metabolism.

Our studies in cultured human muscle cells indicated that exposure to ME/CFS serum led to increased rates of mitochondrial respiration, driven by amino acids alone or in combination with glucose. This effect was particularly evident under conditions of energy depletion, when mitochondrial respiration works at a maximum rate (condition IV). Although lactate production tended to be low under resting conditions, it was excessively induced by energetic strain in muscle cells exposed to ME/CFS serum. These observations are compatible with a metabolic obstruction at the level of $\mathrm{PDH}$, causing increased conversion of pyruvate to lactate, and amplified demand for alternative substrates downstream of PDH. The effects of such PDH dysregulation would conceivably escalate significantly under conditions of increased metabolic flux, in accordance with the cell studies reported here. Supporting findings have been demonstrated after knockdown of $\mathrm{PDH}$ in cultured muscle cells (34). Based on these observations, ME/CFS patient serum appears to carry substance(s) that induce a cellular response to support mitochondrial energy production. In accordance, optimization of mitochondrial respiration could be regarded as a protective response to avoid energy depletion caused by PDH dysfunction. The energetic yield from such metabolic adaptation will, however, depend on the supply of energetic substrates to fuel mitochondrial respiration, which appears to be limited in ME/CFS patients. The present findings suggest that mitochondrial fueling is compromised by reduced flux through $\mathrm{PDH}$, leading to overconsumption of alternative substrates such as amino acids linked to the TCA cycle. Additional studies are required to identify the substance(s) in ME/CFS serum that mediate the effects on cultured muscle cells, which could act directly on the metabolic apparatus or indirectly via signaling factors.

Our data, suggesting that ME/CFS is associated with impaired efficiency of energy metabolism combined with lactate overproduction, may explain why many ME/CFS patients reach anaerobic threshold at a low workload (9-11). This is supported by an individual patient report (35) and our own observations of patients with severe ME/CFS that had lactate levels elevated to more than $8 \mathrm{mM}$ from just standing upright for 10 minutes. Since ME/CFS patients normally do not seem to have increased lactate levels at rest (14), it may be speculated that the metabolism adapts to the putative impaired PDH function by increasing the oxidation of acetyl-CoA-yielding amino acids and perhaps also of fatty acids. Thus, the PDH-dependent metabolism may function sufficiently to avoid lactate overproduction in the resting patients but will not be able to respond adequately to increased glycolysis when the system is challenged by exertion. The TCA cycle flux is estimated to increase by 70 - to 100 -fold during strenuous exercise in order to generate sufficient ATP (36). This would create a situation with insufficient supply of acetyl-CoA to fuel oxidative ATP production as well as accumulation of pyruvate and subsequently lactate, despite aerobic conditions with adequate oxygen levels. Impairment in $\mathrm{PDH}$ function could therefore be consistent with the reduction in aerobic threshold, poor recovery, and postexertional malaise as observed in ME/CFS patients. Such a mechanism would be in agreement with the observed difference in workload intolerance versus disease severity among ME/CFS patients, by relating symptoms to the magnitude of metabolic obstruction.

The finding that PDKs are upregulated in PBMCs in ME/CFS patients is suggestive of metabolic dysregulation at a systemic level. These cells have a functional PPAR-PDK interaction (37) and may therefore mirror associated effects occurring in other tissues that are responsible for the clinical presentation and symptoms of ME/CFS. Importantly, even though the reductions in amino acids levels were highly significant in female ME/CFS patients, the effect sizes were moderate. The serum levels of the acetyl-CoA-producing and anaplerotic amino acids were within normal ranges also in most female ME/ CFS patients. Thus, there are no signs of amino acid deficiency, and there is no obvious need for dietary supplementations. The observed reductions in amino acids in ME/CFS patients may merely reflect an altered metabolism in efforts to adapt to a metabolic obstruction. The pattern of amino acid changes could not be explained by other variables, such as age, level of inactivity assessed by steps per 24 hours, ME/CFS disease severity, or disease duration. Even though there were significant correlations to BMI, all categories of ME/CFS women, including obese women, had lower mean levels of the ketogenic amino acids than healthy control women, i.e., the differences in BMI could not explain the observed reduced serum levels of category II amino acids. We compared serum levels of amino acids between overnight fasting and nonfasting ME/CFS patients and found the same pattern of changes in amino acid profiles with or without 
inclusion of the fasting patients. However, there were significant reductions of several category II amino acids among the overnight fasting patients. Thus, even though the effect of fasting was limited, we excluded the overnight fasting patients from the analyses to avoid this confounding variable.

This study identifies metabolic changes that may explain several of the clinical symptoms in ME/CFS patients; however, the underlying mechanism(s) causing these effects still remains unclear. Our previous findings in interventional clinical trials evaluating B lymphocyte depletion therapy, using the monoclonal anti-CD20 antibody rituximab, suggest that ME/CFS in a subgroup of patients could be a variant of an autoimmune disease (38-40), possibly involving antibodies. Future research should address the metabolic effects in ME/CFS reported in the present study in the context of a possible immunological mechanism. Autoantibodies may potentially interfere with cell surface receptors as well as other factors that are involved in the cascades of cell signaling. A recent study showed that serum levels of autoantibodies to several autonomic receptors were higher in ME/CFS patients than in healthy controls (41). Similar autoantibodies were detected in postural orthostatic tachycardia syndrome (42) and in complex regional pain syndrome (43), conditions with some resemblances to ME/CFS, affecting fertile women after a triggering event $(44,45)$. Likewise, autoantibodies in ME/CFS may interfere with key metabolic signaling pathways involving factors such as HIF1 (24) and PPARs, as well as AMPK, which was abnormally activated in skeletal muscle cells of ME/CFS patients (46). These factors are associated with PDK (22) and SIRT4 (47) in the regulation of oxidative metabolism. Such signaling pathways involve factors previously linked to ME/CFS, such as reactive oxygen species associated with signaling and mitochondrial dysfunction (48), and nitric oxide related to endothelial dysfunction, which has been demonstrated in ME/CFS (27). AMPK activates the endothelial nitric oxide synthase enzyme (eNOS) (49), and corrupted AMPK signaling may be a mechanism in endothelial dysfunction. In the present study, the mean serum level of the endogenous eNOS inhibitor and L-Arg analog ADMA was similar in ME/CFS patients and healthy controls. ADMA is usually elevated in cardiovascular endothelial dysfunction (50), indicating that the endothelial dysfunction mechanisms in ME/CFS, mainly affecting women of fertile age, differ from those in cardiovascular diseases.

$\mathrm{PDH}$ deficiency is demonstrated in severe metabolic disorders caused by mutations, involving progressive neurological degeneration, acidosis, energy deficit, and associations with epilepsy due to altered neuronal excitability (51). In the autoimmune disease primary biliary cirrhosis, which is accompanied by major fatigue, antimitochondrial autoantibodies target $\mathrm{PDH}$ complex components, leading to inflammation and tissue destruction $(52,53)$. In ME/CFS, there is no convincing evidence for histological inflammation or tissue destruction, yet there are data showing activation of both proinflammatory and antiinflammatory pathways in cytokine analyses, especially during the first years of the disease (6). ME/CFS often presents with varying symptoms over time, which are to some extent reversible, sometimes with spontaneous remissions and with observed clinical responses after B cell depletion therapy (38). Further work may focus on how abnormal immune responses in ME/CFS could affect cellular signaling to cause PDH inhibition.

In conclusion, this study suggests that ME/CFS is associated with $\mathrm{PDH}$ impairment, leading to increased consumption of amino acids that fuel alternative pathways for ATP production. ME/CFS patient serum was found to increase mitochondrial respiration in cultured muscle cells, possibly as a compensation or adaptation to an inhibition of metabolic energy pathways. The physiological consequences of such defects are likely to include energy (ATP) deficiency and excessive lactate production, in agreement with the debilitating exertion intolerance seen in ME/CFS patients.

\section{Methods}

$M E / C F S$ patients and healthy controls. All patients participating in this study fulfilled the Canadian consensus criteria for ME/CFS (1). The blood samples were collected before intervention (baseline) in three separate clinical trials. The KTS-2-2010 trial (NCT01156909) was an open-label and one-armed phase II study in Bergen, Norway, for the investigation of rituximab maintenance therapy in 29 ME/CFS patients (38). The "RituxME" trial (NCT02229942) is an ongoing multicenter, randomized, double-blind, and placebo-controlled phase III study in Norway, including 151 patients with ME/CFS, aiming to evaluate previous findings indicating therapeutic benefit of B cell depletion therapy in ME/CFS (38-40). The "CycloME" trial (NCT02444091) is an ongoing, open-label, phase II study in Bergen, evaluating cyclophosphamide treatment in $40 \mathrm{ME} / \mathrm{CFS}$ patients.

The $200 \mathrm{ME} / \mathrm{CFS}$ patients in the present study included 162 women and 38 men. The mean age of female ME/CFS patients was 37.8 years (range 18.1-62.2 years). The mean age of the male ME/CFS patients was 
36.3 years (range 18.7-63.9 years). BMI was calculated as $\mathrm{kg} / \mathrm{m}^{2}$. Among female ME/CFS patients, mean BMI was 24.9 (range 16.6-41.8). In male ME/CFS patients, mean BMI was 25.0 (range 17.4-33.5). 67 women and 35 men constituted the 102 healthy controls, with no chronic disease or chronic medication. Among these, 62 healthy controls were recruited from blood donors at Haukeland University Hospital, with blood samples taken in 2012. 40 healthy controls were recruited from the staff at the Department of Oncology, Haukeland University Hospital, with blood samples taken in 2015. The healthy women were of a mean age of 34.5 years (range 19.0-57.0 years), and the healthy men were of a mean age of 36.2 years (range 21.0-65.0 years).

Baseline blood samples from ME/CFS patients and healthy individuals were collected by venous puncture. All samples were immediately processed according to a standardized biobank procedure in the trial protocols, and serum samples were stored at $-80^{\circ} \mathrm{C}$ prior to analyses.

Among the $200 \mathrm{ME} / \mathrm{CFS}$ patients, 47 fasted overnight prior to biobank sampling, while 153 did not. All 102 healthy controls were nonfasting. We compared serum concentrations of all amino acids, between overnight fasting and nonfasting patients, by sex (Supplemental Tables 1 and 2). Further statistical analyses of serum amino acid concentrations were performed, comparing only the nonfasting ME/ CFS patients and healthy controls. The overnight fasting patients were kept separate in the data analyses to avoid a confounding effect.

Metabolite analysis. The 20 standard amino acids were analyzed by robotic workstations using gas chromatography-tandem mass spectrometry $(54,55)$, with within and between day CVs of $2 \%-5 \%$. Arg, hArg, ADMA, SDMA, 1-MHis, and 3-MHis were analyzed by liquid chromatography-tandem mass spectrometry (56), with within and between day CVs of 3\%-12\%. Measurements of metabolite concentrations, including amino acids, generally tend to be higher in serum compared with plasma samples, but there is an overall correlation reflecting that changes in the metabolic profile are proportionally displayed in the two matrices (57). The majority of samples from ME/CFS patients were harvested in late 2014 and 2015 (181 samples from the "RituxME" and "CycloME" trials). The remaining 19 samples were collected in 2010 (in the KTS-2-2010 trial). Of the 102 serum samples from healthy controls, 62 were collected in 2012 and 40 were collected in 2015. Details for analyses of sample storage of each metabolite are described in the Supplemental Methods.

Quantitative RT-PCR. All ME/CFS patients and healthy controls included in the gene expression study were nonfasting at blood sampling. First, all TaqMan assays (Applied Biosystems) were performed in 21 ME/CFS patients and 21 healthy controls, testing external $\beta$-actin (ACTB, assay Hs99999903_m1), external GAPDH (assay Hs99999905_m1), or internal coamplified $\beta$-actin (ACTB, assay Hs01060665_m1) for normalization. Duplex qRT-PCR assays, with target gene and coamplified $A C T B$ as internal control, had the best performance, with low variation among triplicates, and were used for assessment of $P D K 1, P D K 2, P D K 3, P D K 4$, PPARA, PPARD, PPARG, PDHA, MPC1, MPC2, ACOX1, HIF1A, and SIRT4 mRNAs in $75 \mathrm{ME} / \mathrm{CFS}$ patients and 43 healthy controls. All qRT-PCR assays were run with triplicates for each sample, with all samples on the same plate. For all genes except PPARG and SIRT4, the median SD among triplicates for the 118 samples was in the range of $6 \%-8 \%$. The mRNA expression level of SIRT4 in PBMCs was low, and samples with SD $\geq 30 \%$ were excluded (11 of $75 \mathrm{ME} / \mathrm{CFS}$ patients, 5 of 43 healthy controls), giving a mean SD for triplicates of $12 \%$ in both groups. For PPARG qRT-PCR, the expression level was below the detection limit. Details regarding total RNA extraction, cDNA synthesis, and qRT-PCR and the TaqMan assays used to assess the respective mRNA expression levels of the genes in PBMCs are shown in the Supplemental Methods.

Cell culture. HSMM (Lonza) were maintained in accordance with standard cell culture protocols in SkGM-2 medium (Lonza) with growth factors and supplements as recommended by the supplier. The experiments were performed using cells that had been passaged for fewer than 10 population doublings. To investigate if exposure to ME/CFS patient serum influenced energy metabolism in cultured myoblasts, fetal bovine serum was replaced by serum (20\%) from healthy individuals (controls) or from ME/CFS patients in our cohort. The cells were seeded at a density of 8,000 cells per well in a 96-well plate. The cells were then incubated at least 18 hours before exposure to control or patient serum was initiated. The exposure time was up to 6 days, and the culture medium was routinely refreshed at day 3 . The experiments were performed blinded regarding group affiliation (ME/CFS or healthy control).

Cellular metabolism. OCR and ECAR were measured to assess rates of mitochondrial respiration and lactate production (glycolysis), respectively (Seahorse XFe96 Analyzer, Seahorse Bioscience). The measurements were performed in 96-well HSMM cultures and involved established protocols used to obtain specific metabolic parameters (58). The assay conditions were optimized with regards to cell number and 
concentrations of CCCP and oligomycin. Following incubation with control or patient serum, the growth medium was replaced with unbuffered assay medium (DMEM, Sigma-Aldrich; $\mathrm{pH}$ 7.4; without glucose, L-Gln, phenol red, sodium pyruvate, and sodium bicarbonate). The cells were placed at $37^{\circ} \mathrm{C}$ in a $\mathrm{CO}_{2}$-free incubator (Seahorse XF Prep station) for 1 to 1.5 hours, before they were transferred to the analyzer. OCR and ECAR were simultaneously recorded throughout the experiment, which involved sequential administrations of specific metabolic modulators. Unless otherwise stated, the treatment series (injection) was glucose $(10 \mathrm{mM})$, oligomycin $(3 \mu \mathrm{M}), \mathrm{CCCP}(2 \mu \mathrm{M})$, and then rotenone. The residual OCR after rotenone/ antimycin A treatment was subtracted as background from the other OCR measurements. The implications of the different chemical modulators are further described below and in legends to Figure 5. After the analysis, protein content was measured using the Pierce BCA Protein Assay Kit (ThermoFisher Scientific) to confirm that the cellular amount was similar in cultures exposed to serum from ME/CFS patients compared with serum from healthy individuals.

The following cellular energetic conditions were defined. For condition I "Resting (amino acids)," the only energy substrates present were amino acids (not Gln) provided in the assay medium. The cells were in resting mode since they only need energy to maintain basal physiological requirements. For condition II "Resting (with amino acids and glucose)," the cells were provided glucose, but they were still in energetic resting mode since the demand for ATP was not changed. For condition III "Anaerobic strain," ATP synthase was inhibited by oligomycin, and the cells relied strictly on glycolytic ATP production. This is similar to anaerobic energy metabolism, in which mitochondrial ATP production is inhibited by oxygen depletion. Under these conditions, the maximal glycolytic capacity was measured. The associated OCR is called leak activity, since it is not linked to ATP production via oxidative phosphorylation. A low rate of leak respiration means that the integrity of the mitochondrial oxidative phosphorylation system is intact. For condition IV "Aerobic strain," addition of CCCP released the inhibitory effect of oligomycin on mitochondrial oxygen consumption by dissipating the mitochondrial membrane potential and thereby uncoupling the electron transport system from ATP production. This reflects a situation of severe energy depletion, in which both mitochondrial respiration and glycolysis work at maximal flux. Under this condition, the maximal capacity of mitochondrial oxygen consumption was measured. Finally, rotenone and antimycin A were administered to inhibit respiratory complex I and III, respectively, in order to assess nonmitochondrial oxygen consumption (subtracted as OCR background).

Statistics. Statistical analyses were performed using SPSS version 23 and Graphpad prism version 7.0. In comparisons of mean serum levels of amino acids between ME/CFS patients and healthy controls, unpaired 2-tailed $t$ tests were used (equal variances not assumed). For analyses comparing ME/CFS patients and healthy controls by sex, ANOVA (adjusted $P$ values) were used. For 1-MHis, Mann-Whitney $U$ test for independent samples or Kruskal-Wallis test were used. Effect sizes for differences significant by 2 -tailed $t$ tests were assessed by Cohen's $d$. For comparison of mean serum levels of amino acids versus categories of BMI, ME/CFS severity, ME/CFS duration, activity levels (steps per 24 hours), and SF36mean5 score, both for difference between categories and for linearity, $P$ values (adjusted) were from ANOVA analyses (or Kruskal-Wallis for 1-MHis).

Due to the lower number of male ME/CFS patients (29 nonfasting) included for amino acid analyses, compared with 124 nonfasting female ME/CFS patients, the statistical strength to detect a difference was lower among men. To avoid possible misinterpretation regarding sex differences, separate analyses were performed where two nonoverlapping groups of 33 female patients were randomly selected from the 124 nonfasting female ME/CFS patients, and two nonoverlapping groups of 33 healthy women selected from the 67 nonfasting healthy women. We compared the sums of serum concentrations of category II and category III amino acids by 2-tailed $t$ tests. There were significantly lower category II amino acid levels in 33 female ME/CFS patients versus 33 healthy controls in both data sets $(P=0.022$ and $P=0.002$, respective1y). In addition, the sum of serum concentrations of category III (anaplerotic) amino acids was significantly lower in 33 female ME/CFS patients compared with 33 healthy women in both data sets $(P=0.021$ and $P=0.001$, respectively). In comparison, there were no significant differences between 29 nonfasting male $\mathrm{ME} / \mathrm{CFS}$ patients and 35 healthy men for the sums of category II or category III amino acids.

The present study is explorative and hypothesis generating, comparing ME/CFS patients versus healthy controls. For the 26 amino acids analyzed, no adjustments for multiple comparisons were performed, except for adjusted analyses in the ANOVA and Kruskal-Wallis tests used to compare subgroups. The observed differences between patients and healthy controls, for each amino acid and for the sum of 
amino acids within a category, were in general similar. Thus, amino acids within a category showed the same pattern of changes. Correlations between serum levels of amino acids, and between serum levels of amino acids and clinical variables, were performed using Pearson's analyses. Mann-Whitney $U$ tests for independent samples were performed for comparison of TaqMan qRT-PCR data (relative mRNA levels) between ME/CFS patients and healthy controls, and Kruskal-Wallis tests were used for analyses of differences in mRNA levels between categories of clinical variables. Correlation analyses among mRNA data for different genes were performed using Spearman's analyses. For comparisons of data from OCR and ECAR analyses of cell cultures exposed to serum from either ME/CFS patients or healthy controls, Mann-Whitney $U$ tests were used. All $P$ values were two-sided. $P$ values of less than 0.05 were considered significant.

Study approval. The clinical trials from which biobank samples were used in this study, including blood samples from healthy controls, were approved by the Regional Ethical Committee (Tromsø, Norway), no. 2010/1318-4, no. 2014/365, and no. 2014/1672. All patients provided written informed consent.

\section{Author contributions}

$\varnothing \mathrm{F}, \mathrm{OM}, \mathrm{OB}$, and KJT designed the study. ØF, OM, IGR, AF, IKV, K Sørland, KL, IH, HT, MEG, KAB, LMLJB, and CS included patients in clinical studies and provided biobank samples. OB, KR, DS, KA, and SL performed laboratory experiments. SED, GVR, and KJT performed the cell culture experiments. PMU and AM measured serum amino acids. PMU, AM, AF, KL, K Sommerfelt, LH, and OD provided scientific and technical advice. $\varnothing \mathrm{F}, \mathrm{OB}$, and $\mathrm{KJT}$ did the data analyses. $\varnothing \mathrm{F}, \mathrm{KJT}$, and OM wrote the paper. All authors approved the final manuscript.

\section{Acknowledgments}

The authors thank the study personnel at each center of the "RituxME" and "CycloME" clinical trials for patient follow-up and biobank sampling. This work has received financial support from The Kavli Trust. The clinical trials recruiting patients for the biobank and the laboratory analysis in this study received financial support from the Research Council of Norway, the Norwegian Regional Health Trusts, the MEandYou Foundation, the Norwegian ME Association, and from the legacy of Torstein Hereid. These funders had no role in trial design, data collection, analysis, decision to publish, or preparation of the manuscript.

Address correspondence to: Øystein Fluge, Department of Oncology and Medical Physics, Haukeland University Hospital, Jonas Lies vei 65, N-5021 Bergen, Norway. Phone: 47.55973587; E-mail: oystein. fluge@helse-bergen.no.

1. Carruthers BM, et al. Myalgic encephalomyelitis/ chronic fatigue syndrome: clinical working case definition, diagnostic and treatment protocols. J Chronic Fatigue Syndr. 2003;11(1):7-36.

2. Nacul LC, et al. The functional status and well being of people with myalgic encephalomyelitis/chronic fatigue syndrome and their carers. BMC Public Health. 2011;11:402.

3. Falk Hvidberg M, Brinth LS, Olesen AV, Petersen KD, Ehlers L. The health-related quality of life for patients with myalgic encephalomyelitis/chronic fatigue syndrome (ME/CFS). PLoS One. 2015;10(7):e0132421.

4. Armstrong CW, McGregor NR, Sheedy JR, Buttfield I, Butt HL, Gooley PR. NMR metabolic profiling of serum identifies amino acid disturbances in chronic fatigue syndrome. Clin Chim Acta. 2012;413(19-20):1525-1531.

5. Filler K, et al. Association of Mitochondrial Dysfunction and Fatigue: A Review of the Literature. BBA Clin. 2014;1:12-23.

6. Hornig M, et al. Distinct plasma immune signatures in ME/CFS are present early in the course of illness. Sci Adv. 2015; 1(1):1.

7. Morris G, Maes M. A neuro-immune model of myalgic encephalomyelitis/chronic fatigue syndrome. Metab Brain Dis. 2013;28(4):523-540.

8. Rowe PC, et al. Neuromuscular strain increases symptom intensity in chronic fatigue syndrome. PLoS ONE. 2016;11(7):e0159386.

9. Vermeulen RC, Vermeulen van Eck IW. Decreased oxygen extraction during cardiopulmonary exercise test in patients with chronic fatigue syndrome. J Transl Med. 2014;12:20.

10. Snell CR, Stevens SR, Davenport TE, Van Ness JM. Discriminative validity of metabolic and workload measurements for identifying people with chronic fatigue syndrome. Phys Ther. 2013;93(11):1484-1492.

11. Keller BA, Pryor JL, Giloteaux L. Inability of myalgic encephalomyelitis/chronic fatigue syndrome patients to reproduce $\mathrm{VO}_{2}$ peak indicates functional impairment. J Transl Med. 2014;12:104.

12. Murrough JW, et al. Increased ventricular lactate in chronic fatigue syndrome measured by $1 \mathrm{H}$ MRS imaging at $3.0 \mathrm{~T}$. II: comparison with major depressive disorder. NMR Biomed. 2010;23(6):643-650.

13. Shungu DC, et al. Increased ventricular lactate in chronic fatigue syndrome. III. Relationships to cortical glutathione and clinical symptoms implicate oxidative stress in disorder pathophysiology. NMR Biomed. 2012;25(9):1073-1087.

14. Armstrong CW, McGregor NR, Lewis DP, Butt HL, Gooley PR. Metabolic profiling reveals anomalous energy metabolism and 
oxidative stress pathways in chronic fatigue syndrome patients. Metabolomics. 2015;11(6):1626-1639.

15. Niblett SH, et al. Hematologic and urinary excretion anomalies in patients with chronic fatigue syndrome. Exp Biol Med (Maywood). 2007;232(8):1041-1049.

16. Robinson BH. Lactic acidemia and mitochondrial disease. Mol Genet Metab. 2006;89(1-2):3-13.

17. Myhill S, Booth NE, McLaren-Howard J. Chronic fatigue syndrome and mitochondrial dysfunction. Int J Clin Exp Med. 2009;2(1):1-16.

18. Patel MS, Nemeria NS, Furey W, Jordan F. The pyruvate dehydrogenase complexes: structure-based function and regulation. $J$ Biol Chem. 2014;289(24):16615-16623.

19. Kolobova E, Tuganova A, Boulatnikov I, Popov KM. Regulation of pyruvate dehydrogenase activity through phosphorylation at multiple sites. Biochem J. 2001;358(Pt 1):69-77.

20. Zhang S, Hulver MW, McMillan RP, Cline MA, Gilbert ER. The pivotal role of pyruvate dehydrogenase kinases in metabolic flexibility. Nutr Metab (Lond). 2014;11(1):10.

21. Mathias RA, et al. Sirtuin 4 is a lipoamidase regulating pyruvate dehydrogenase complex activity. Cell. 2014;159(7):1615-1625.

22. Fritzen AM, et al. 5'-AMP activated protein kinase $\alpha 2$ controls substrate metabolism during post-exercise recovery via regulation of pyruvate dehydrogenase kinase 4. J Physiol (Lond). 2015;593(21):4765-4780.

23. Nakamura MT, Yudell BE, Loor JJ. Regulation of energy metabolism by long-chain fatty acids. Prog Lipid Res. 2014;53:124144.

24. Kim JW, Tchernyshyov I, Semenza GL, Dang CV. HIF-1-mediated expression of pyruvate dehydrogenase kinase: a metabolic switch required for cellular adaptation to hypoxia. Cell Metab. 2006;3(3):177-185.

25. Rutherford G, Manning P, Newton JL. Understanding muscle dysfunction in chronic fatigue syndrome. J Aging Res. 2016;2016:2497348

26. Berg JM, Tymoczko JL, Gatto GJ, Stryer L. Biochemistry. New York, NY: W. H. Freeman; 2015.

27. Newton DJ, Kennedy G, Chan KK, Lang CC, Belch JJ, Khan F. Large and small artery endothelial dysfunction in chronic fatigue syndrome. Int J Cardiol. 2012;154(3):335-336.

28. Dragsted LO. Biomarkers of meat intake and the application of nutrigenomics. Meat Sci. 2010;84(2):301-307.

29. Aquilani R, et al. Myofibrillar protein overdegradation in overweight patients with chronic heart failure: the relationship to serum potassium levels. Nutrition. 2014;30(4):436-439.

30. Yamano E, et al. Index markers of chronic fatigue syndrome with dysfunction of TCA and urea cycles. Sci Rep. 2016;6:34990

31. Bender T, Martinou JC. The mitochondrial pyruvate carrier in health and disease: To carry or not to carry? Biochim Biophys Acta. 2016;1863(10):2436-2442.

32. Naviaux RK, et al. Metabolic features of chronic fatigue syndrome. Proc Natl Acad Sci USA. 2016;113(37):E5472-E5480.

33. Krumsiek J, et al. Gender-specific pathway differences in the human serum metabolome. Metabolomics. 2015;11(6):1815-1833.

34. Nogiec C, Burkart A, Dreyfuss JM, Lerin C, Kasif S, Patti ME. Metabolic modeling of muscle metabolism identifies key reactions linked to insulin resistance phenotypes. Mol Metab. 2015;4(3):151-163.

35. Vink M. The aerobic energy production and the lactate excretion are both impeded in myalgic encephalopathy/chronic fatigue syndrome. J Neurol Neurobiol. 2015;1:4.

36. Gibala MJ, MacLean DA, Graham TE, Saltin B. Tricarboxylic acid cycle intermediate pool size and estimated cycle flux in human muscle during exercise. Am J Physiol. 1998;275(2 Pt 1):E235-E242.

37. Bouwens M, Afman LA, Müller M. Fasting induces changes in peripheral blood mononuclear cell gene expression profiles related to increases in fatty acid beta-oxidation: functional role of peroxisome proliferator activated receptor alpha in human peripheral blood mononuclear cells. Am J Clin Nutr. 2007;86(5):1515-1523.

38. Fluge Ø, et al. B-Lymphocyte Depletion in Myalgic Encephalopathy/Chronic Fatigue Syndrome. An Open-Label Phase II Study with Rituximab Maintenance Treatment. PLoS ONE. 2015;10(7):e0129898.

39. Fluge $\varnothing$, et al. Benefit from B-lymphocyte depletion using the anti-CD20 antibody rituximab in chronic fatigue syndrome. A double-blind and placebo-controlled study. PLoS One. 2011;6(10):e26358.

40. Fluge $\varnothing$, Mella O. Clinical impact of B-cell depletion with the anti-CD20 antibody rituximab in chronic fatigue syndrome: a preliminary case series. BMC Neurol. 2009;9:28

41. Loebel M, et al. Antibodies to $\beta$ adrenergic and muscarinic cholinergic receptors in patients with Chronic Fatigue Syndrome. Brain Behav Immun. 2016;52:32-39.

42. Li H, et al. Autoimmune basis for postural tachycardia syndrome. J Am Heart Assoc. 2014;3(1):e000755.

43. Kohr D, et al. Autoimmunity against the $\beta 2$ adrenergic receptor and muscarinic- 2 receptor in complex regional pain syndrome. Pain. 2011;152(12):2690-2700.

44. Dahan S, Tomljenovic L, Shoenfeld Y. Postural Orthostatic Tachycardia Syndrome (POTS)--A novel member of the autoimmune family. Lupus. 2016;25(4):339-342.

45. Goebel A, Blaes F. Complex regional pain syndrome, prototype of a novel kind of autoimmune disease. Autoimmun Rev. 2013;12(6):682-686.

46. Brown AE, Jones DE, Walker M, Newton JL. Abnormalities of AMPK activation and glucose uptake in cultured skeletal muscle cells from individuals with chronic fatigue syndrome. PLoS One. 2015;10(4):e0122982.

47. Mathias RA, Greco TM, Cristea IM. Identification of sirtuin4 (SIRT4) protein interactions: uncovering candidate acyl-modified mitochondrial substrates and enzymatic regulators. Methods Mol Biol. 2016;1436:213-239.

48. Morris G, Maes M. Mitochondrial dysfunctions in myalgic encephalomyelitis/chronic fatigue syndrome explained by activated immuno-inflammatory, oxidative and nitrosative stress pathways. Metab Brain Dis. 2014;29(1):19-36.

49. Schulz E, Schuhmacher S, Münzel T. When metabolism rules perfusion: AMPK-mediated endothelial nitric oxide synthase activation. Circ Res. 2009;104(4):422-424.

50. Sibal L, Agarwal SC, Home PD, Boger RH. The role of asymmetric dimethylarginine (ADMA) in endothelial dysfunction and cardiovascular disease. Curr Cardiol Rev. 2010;6(2):82-90

51. Bhandary S, Aguan K. Pyruvate dehydrogenase complex deficiency and its relationship with epilepsy frequency--An overview. Epilepsy Res. 2015;116:40-52. 
52. Yamagiwa S, Kamimura H, Takamura M, Aoyagi Y. Autoantibodies in primary biliary cirrhosis: recent progress in research on the pathogenetic and clinical significance. World J Gastroenterol. 2014;20(10):2606-2612.

53. Hollingsworth KG, Newton JL, Robinson L, Taylor R, Blamire AM, Jones DE. Loss of capacity to recover from acidosis in repeat exercise is strongly associated with fatigue in primary biliary cirrhosis. J Hepatol. 2010;53(1):155-161.

54. Windelberg A, Arseth O, Kvalheim G, Ueland PM. Automated assay for the determination of methylmalonic acid, total homocysteine, and related amino acids in human serum or plasma by means of methylchloroformate derivatization and gas chromatography-mass spectrometry. Clin Chem. 2005;51(11):2103-2109.

55. Ueland PM, Midttun O, Windelberg A, Svardal A, Skålevik R, Hustad S. Quantitative profiling of folate and one-carbon metabolism in large-scale epidemiological studies by mass spectrometry. Clin Chem Lab Med. 2007;45(12):1737-1745.

56. Midttun $\varnothing$, Kvalheim G, Ueland PM. High-throughput, low-volume, multianalyte quantification of plasma metabolites related to one-carbon metabolism using HPLC-MS/MS. Anal Bioanal Chem. 2013;405(6):2009-2017.

57. Yu Z, et al. Differences between human plasma and serum metabolite profiles. PLoS One. 2011;6(7):e21230.

58. VanLinden MR, et al. Subcellular distribution of NAD+ between cytosol and mitochondria determines the metabolic profile of human cells. J Biol Chem. 2015;290(46):27644-27659. 